\title{
An assessment of marine atmospheric boundary layer roll detection using Sentinel-1 SAR data
}

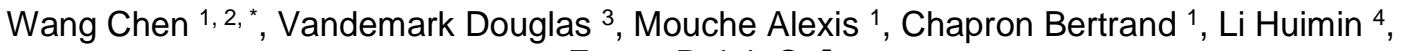 \\ Foster Ralph C. ${ }^{5}$
}

${ }^{1}$ IFREMER, Univ. Brest, CNRS, IRD, Laboratoire d'Oceanographie Physique et Spatiale (LOPS), Brest, France

2 IMT Atlantique, Lab-STICC, UBL, Brest, France

${ }^{3}$ Ocean Processes Analysis Laboratory, University of New Hampshire, Durham, NH, USA

${ }^{4}$ School of Marine Sciences, Nanjing University of Information Science and Technology, Nanjing, China

${ }^{5}$ Applied Physics Laboratory, University of Washington, Seattle, WA, USA

*Corresponding author : Chen Wang, email address : $\underline{\text { Chen.Wang@ifremer.fr }}$

\begin{abstract}
:
The ability of high-resolution synthetic aperture radar (SAR) to detect marine atmospheric boundary layer (MABL) roll-induced roughness modulation of the sea surface wave field is well known. This study presents SAR measurements of MABL rolls using global coverage data collected by the European Space Agency's C-band Sentinel-1A satellite in 2016-2017. An automated classifier is used to identify likely roll events from more than 1.3 million images that were acquired at two incidence angles of $23^{\circ}$ and $36.5^{\circ}$ in either VV or $\mathrm{HH}$ polarization. Characteristics of the detected rolls are examined for different wind speeds, polarizations, incidence and relative azimuth angles. Roll detection counts are much higher at the higher incidence angle and nearly equivalent for VV and $\mathrm{HH}$ polarizations. Detection depends strongly on the relative azimuth with roll detection rates at crosswind being 3-10 times lower than for up- or downwind. All data show a low wind speed threshold near $2 \mathrm{~m} \mathrm{~s}-1$ and that rolls are most commonly observed at wind speeds near $9 \mathrm{~m} \mathrm{~s}-1$. For all viewing configurations, we find that rolls induce a wide range of mean surface wind speed modulation with the most frequent value being $8 \%( \pm 3.5 \%)$. Roll detection at crosswind is associated with stronger roll-induced surface wind enhancement. Dependencies of roll detection on the incidence and relative azimuth angles are consistent with rapid short-scale wind-wave adjustments to the roll-induced surface wind gusts. These $\mathrm{cm}$-scale waves are highly directional and provide limited crosswind backscatter at shallower incidence angles. The same roll-induced surface forcing is thus not equally detectable at all viewing geometries or polarizaions. Stronger and possibly longer-duration wind forcing is likely needed to produce detectable roll-induced modulations at crosswind.
\end{abstract}




\section{Highlights}

- First global statistics of SAR response to MABL rolls using new S-1 WV data. Data show $50 \%$ greater sensitivity to rolls for $36^{\circ}$ vs. $23^{\circ}$ SAR incidence angle. Crosswind (relative to SAR look direction) shows weakest sensitivity to roll imprints. Rolls observed at $23^{\circ}$ and crosswind are more stationary, in more unstable conditions. SAR-estimated surface wind perturbations due to roll impacts are $8 \pm 3.5 \%$.

Keywords : Marine atmospheric boundary layer rolls, Surface wind perturbation, Synthetic aperture radar (SAR), Sentinel-1 wave mode, Imaging sensitivity 
The mean flow in the marine atmospheric boundary layer (MABL) frequently includes an organized secondary circulation in the form of long helical rolls that are approximately aligned along the mean wind direction. They are sometimes made visible by the low-level cloud streets that form in the organized updrafts between rolls (Weston, 1980; Hein and Brown, 1988; Rowe and Houze, 2015). However, rolls are a common feature of the MABL when shear production of turbulence plays an important role and are thus frequently present in the absence of clouds. Investigation of the phenomenon extends back decades, including field, theoretical, numerical, and experimental efforts (Kuettner, 1959; LeMone, 1973; Brown, 1980; Etling and Brown, 1993; Atkinson and Wu Zhang, 1996; Young et al., 2002), and there is evidence that these coherent structures have a measurable impact on turbulent fluxes of heat and momentum across the MABL (Glendening, 1996; Zhang et al., 2008; Zhu, 2008; Brilouet et al., 2017). This inhomogeneous contribution is seldom included in the standard boundary layer parameterizations used in weather forecast and climate models. This is largely because the process occurs at small horizontal length scales that are within the so-called numerical modeling grey zone of 1-10 km (Shin and Hong, 2013; Bauer et al., 2015). Moreover, because they are often invisible to standard remote or in situ sensors, even basic measures of roll characteristics over the oceans have not been established. The fundamental particulars are the frequency of occurrence, strength, wave length, alignment direction, and formation conditions (Levy, 2001; Weckwerth et al., 1997; Zhao et al., 2016; Atkinson and Wu Zhang, 1996; Young et al., 2002).

Synthetic aperture radar (SAR) ocean imagery is able to resolve the parallel backscatter streaks that are associated with the roll-induced surface wind stress changes in day-and-night and most weather conditions (Gerling, 1986; Alpers and Brümmer, 1994; Young, 2000; Vandemark et al., 2001). Case studies using ocean SAR measurements have been conducted to examine MABL rolls in numerous air-sea investigations (Alpers and Brümmer, 1994; Li et al., 2013; Zhao et al., 2016; Babin et al., 2003; Sikora et al., 2011; Alpers et al., 2016). These applications have been limited in scope and mostly dedicated to coastal regions because wide-swath ocean SAR imagery is not acquired routinely nor globally. But a narrow swath option with nearly global coverage, 
sufficient resolution and scene size has been available from the Sentinel-1 SAR satellites since 2014 (Torres et al., 2012). The SAR Wave Mode (WV) extends a legacy of global ocean surface wave monitoring from previous satellite SAR missions. For the purposes of MABL studies, the two most important differences of S-1 WV compared to the legacy data is the increase in image size to 20 by $20 \mathrm{~km}$, while retaining a high spatial resolution of $5 \mathrm{~m}$ pixels, and the addition of a higher incidence angle sample. The European Space Agency (ESA) currently operates two identical Sentinel-1 (S-1) satellites (A\&B) for Copernicus that routinely collect $\sim 130,000$ images each month over most of the ocean surface. At the time of this writing, more than six million images have been acquired.

A required first step for MABL roll studies using SAR data is event detection (Weckwerth et al., 1997; Young et al., 2008). To date, visual inspection has been used to determine the presence of roll imprints in SAR images (e.g. Levy, 2001; Zhao et al., 2016). Given the large number of S-1 WV scenes, as automated method is required. A machine learning tool for S-1 WV image classification was developed from the Inception-v3 convolutional neural network (CNN) to classify each WV image into one of the ten different geophysical categories (Wang et al., 2019b). Note that this classifier very rarely tags non-roll events as rolls but can miss-categorize roll events into other classes. The present study uses only the images that are classified as roll events. More than 1.3 million WV SAR scenes collected in 2016-2017 were analyzed for the presence of MABL rolls, resulting in $\sim 155,000$ roll cases in total. This far surpasses the largest previous SAR MABL roll study of Levy (2001), for which, 7150 SAR images were examined.

The S-1 WV SAR images are acquired at two fixed incidence angles of $23^{\circ}$ (WV1) and $36.5^{\circ}$ (WV2), and with two transmit and receive linear polarization configurations, VV (default) and $\mathrm{HH}$ (experimental). This provides an opportunity for rigorous evaluation of C-band SAR detection and imaging of MABL rolls for varied wind speeds and radar viewing geometries. These characteristics have received limited attention in most previous SAR-based MABL roll investigations (Alpers and Brümmer, 1994; Young, 2000; Sikora and Ufermann, 2004; Li et al., 2013; Zhao et al., 2016), and in ocean SAR studies where the surface wind direction is inferred from roll imprint analyses (Gerling, 1986; Koch, 2004; Christiansen et al., 2006; Lin et al., 2008; Li and Lehner, 2014; Zecchetto, 2018). Alpers and Brümmer (1994) proposed that SAR backscatter due to the roll-induced wave-roughening can be interpreted using the empirical geophysical model functions (GMFs) that relate $20-40 \mathrm{~km}$ scale radar scatterometer measurements to surface wind speed and radar viewing geometry. However, field measurements have shown that surface wind-wave and radar backscatter changes during roll impacts are associated with short-duration and short length-scale wind forcing 
(Vandemark et al., 2001). These roll-induced forces primarily affect the shortest and highly directional wind waves, and not the whole spectrum of surface waves (LeMone, 1973; Lemone, 1976; Young, 2000; Mourad et al., 2000; Vandemark et al., 2001). This interpretation is used to explain the results regarding MABL roll detection and sea surface modulation using the global S-1 WV SAR data that are presented in this paper, and to explain the differences between WV observations and a GMF applied to these observations.

The paper is organized as follows. Data and methods are described in Section 2, including the S-1 WV SAR data, supporting surface environmental variables, and a description of the methods used to classify WV images and to estimate the radar backscatter modulation related to roll-induced wind perturbations. Statistics of the identified MABL roll events are given in Section 3. Section 4 provides analyses of the extracted roll modulation parameters, and compared them to a GMF simulation. Discussion and conclusions follow in Section 5.

\section{Data and methods}

\subsection{Sentinel-1 WV}

Sentinel-1 is a polar-orbiting, sun-synchronous SAR satellite constellation mission designed for long-term operation extending into the next decades. Currently, two satellites (A\&B), which share the same orbital plane offset by a $180^{\circ}$ phase difference, were launched in April of 2014 and 2016, respectively (Torres et al., 2012). The satellites are equipped with identical C-band SAR instruments that operate in four pre-programmed imaging modes: Interferometric Wide swath, Extra Wide swath, Strip Map and WaVe mode (WV). WV is the default mode over the world's ocean except in the Arctic, closed seas and coastal areas, or when S-1 has not been programmed to one of the other imaging modes. There is no WV data acquisition over land except the Amazon rainforest for calibration purposes. WV acquires small SAR image scenes (termed imagettes) at alternating incidence angles of $23^{\circ}$ (WV1) and $36.5^{\circ}$ (WV2). Both usually operate in linear vertical (VV) transmit and receive polarization and, during special phases, in horizontal (HH) polarization. Each WV image size is $20 \mathrm{~km}$ by $20 \mathrm{~km}$, with $5 \mathrm{~m}$ pixel resolution. Neighboring images are spaced by $100-120 \mathrm{~km}$. The orbital repeat cycle is 12 days. Approximately 65,000 imagettes per month are collected by each satellite. The S-1 WV SAR data used in this paper are the Level-1 Single Look Complex (SLC) repository, which are managed at IFREMER (http://www . ifremer . $\mathrm{fr} /$ datavore/exp/dvor/\#/s1quicklook), and are also freely available at ESA's Sentinel Open Access Hub (https://sentinel.esa.int/web/sentinel/sentinel-data-access). 
This study uses S-1A WV SAR data in VV polarization spanning 2016-2017, and S-1B WV data in $\mathrm{HH}$ polarization from 15 March to 01 July 2017. Inland, near-coastal and poleward of $55^{\circ}$ to avoid possible sea ice images are removed. Fig. 1 illustrates the sample population for the VV and $\mathrm{HH}$ datasets on $5^{\circ}$ by $5^{\circ}$ global spatial grid. The typical S- $1 \mathrm{WV}$ coverage is nearly complete over the Pacific, Indian and south Atlantic oceans. There is partial coverage for the eastern north Atlantic because, by default, other standard imaging modes are in operation. The total numbers of image evaluated from these S-1A and S-1B datasets are 1,182,540 and 197,442, respectively.

Each image is co-located in time and space with surface variables from the ERA5 hindcast model, including 10 meter wind components, sea surface temperature, 2 meter air temperature, 2 meter dew-point temperature and surface pressure. ERA5 is the latest generation ECMWF reanalysis product and provides these environmental variables hourly on a global spatial grid of $0.25^{\circ}$ by $0.25^{\circ}$. The data are publicly available at: https://cds.climate.copernicus.eu. From these variables, we estimate the bulk Richardson number $R i_{B}$ at 10 meter height, using the COARE 3.0 air-sea flux algorithm (Fairall et al., 2003).
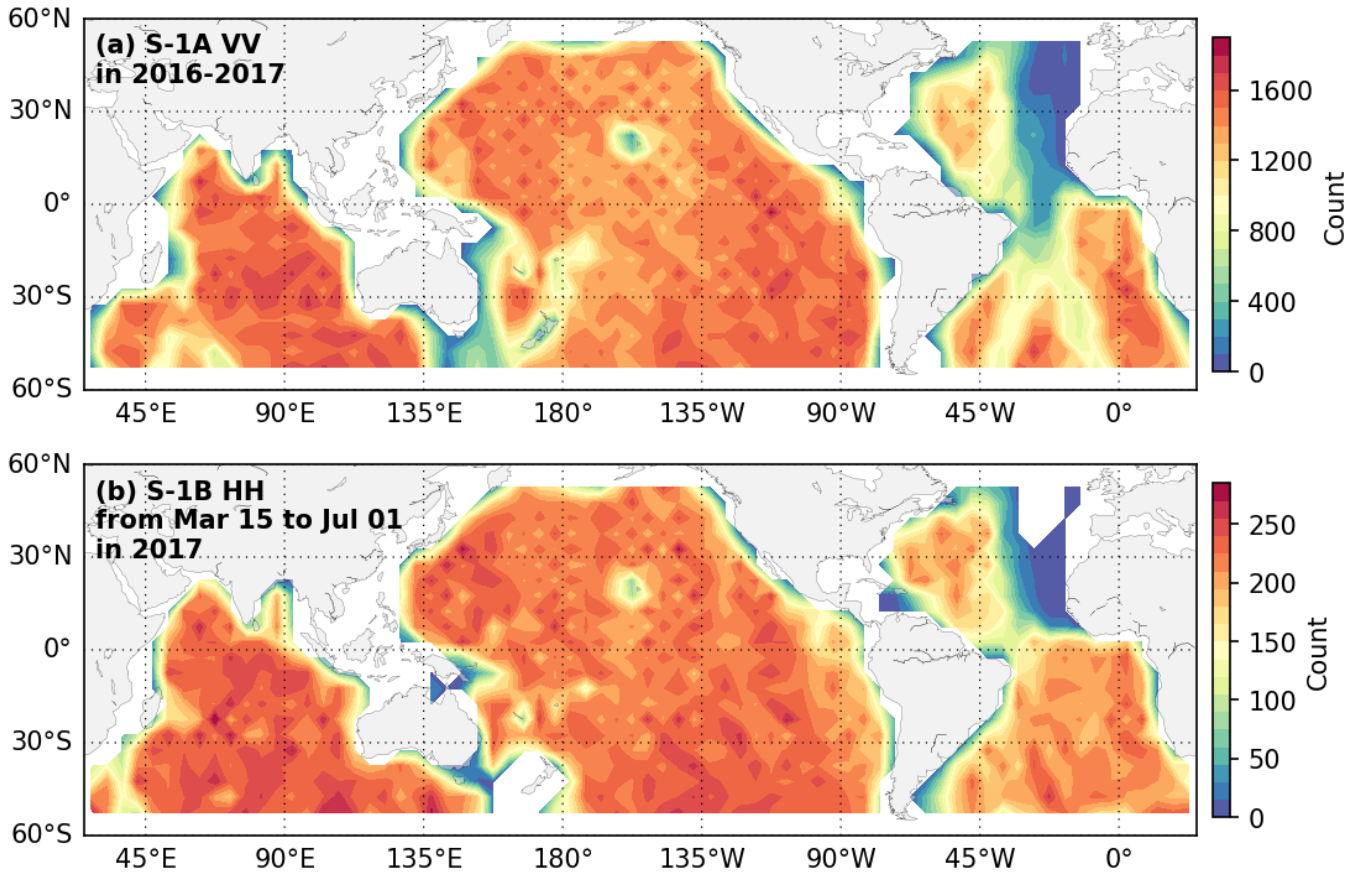

Fig. 1. Ocean SAR data coverage for S-1 study datasets, (a) S-1A in VV polarization in 2016-2017 and (b) S-1B in HH polarization, from 15 Mar to $01 \mathrm{Jul}$ in 2017. Color denotes the number of WV images within each $5^{\circ}$ by $5^{\circ}$ spatial bin. The total number of imagettes in these datasets is $1,182,540$ and 197,442, respectively. 


\subsection{Automated $W V$ image classification}

Oceanic SAR images have been used to identify numerous oceanic, atmospheric, and sea ice features (e.g. Jackson et al., 2004; Wang et al., 2019a,b). The automated image classifier method applied to this WV data catalogue is briefly detailed here, with an emphasis on MABL roll identification (Wang et al., 2019a,b). We first defined the ten most commonly observed geophysical phenomena in the WV image data (Wang et al., 2019a). These phenomena were ocean swell, wind streaks (induced by MABL rolls), micro-convective cells, rain cells, biological slicks, sea ice, icebergs, low wind areas, atmospheric fronts, and oceanic fronts. Visual selection was used to build a large representative collection for each class, leading to an open-access labelled database called TenGeoP-SARwv (Wang et al., 2018). We then developed an automated classification tool based on a deep learning pattern recognition approach. The tool, namely CMwv, was created by fine-tuning the Inception-v3 deep convolutional neural network (CNN) to discriminate between the ten input training sets (Szegedy et al., 2016; Wang et al., 2019b). Separate CMwv models were built for VV-pol WV1 and WV2 data, which are applicable for both S-1A and S-1B WV SAR data. Although the model skill has only been formally evaluated for the VV-polarized SAR images, results suggested that it performs similarly for the HH SAR data, at least for the task of MABL roll identification and analyses presented in this study.

CMwv assigns each WV image probability scores for the ten pre-defined classes. These probabilities add up to 1, and a WV image is considered to represent a case of visually-distinct MABL roll impacts (i.e. wind streaks) if the roll class score is the largest among the ten. The quantified skill for this approach has a Recall (sensitivity) of $83 \%$ for both modes (WV1 and WV2), and Precision (positive detection rate) of $77 \%$ and $96 \%$ for WV1 and WV2, respectively (Wang et al., 2019b). The precision difference between WV1 and WV2 is likely due to a weaker MABL roll imprint in WV1 images, which is one focus of this study. Wang et al. (2019a,b) documented that image contrasts due to roll imprints for WV2 were qualitatively stronger than for WV1 during the visual labelling procedure. Specific to the CMwv machine learning approach, the ability of this deep CNN model to differentiate between phenomena in each SAR image relies on the efficient extraction of optimal features into convolutional layers, and then to amplify feature differences through pooled layers (LeCun et al., 2015; Zhang et al., 2016). That is, distinct image features cannot be extracted if roll imprints are insufficiently clear. Even with these caveats, the overall CMwv precision scores are high. Potential study limitations due to the classification model are discussed in section 5 . 


\subsection{Extraction of roll-induced backscatter modulation amplitude and direction}

Prior to estimation of roll-induced SAR backscatter modulation for varying wind conditions, an objective SAR backscatter recalibration method is used to correct S-1 SAR normalized radar crosssection (NRCS, $\sigma^{0}$ ) as described in Li et al. (2019b). Specific details are provided in Appendix A.
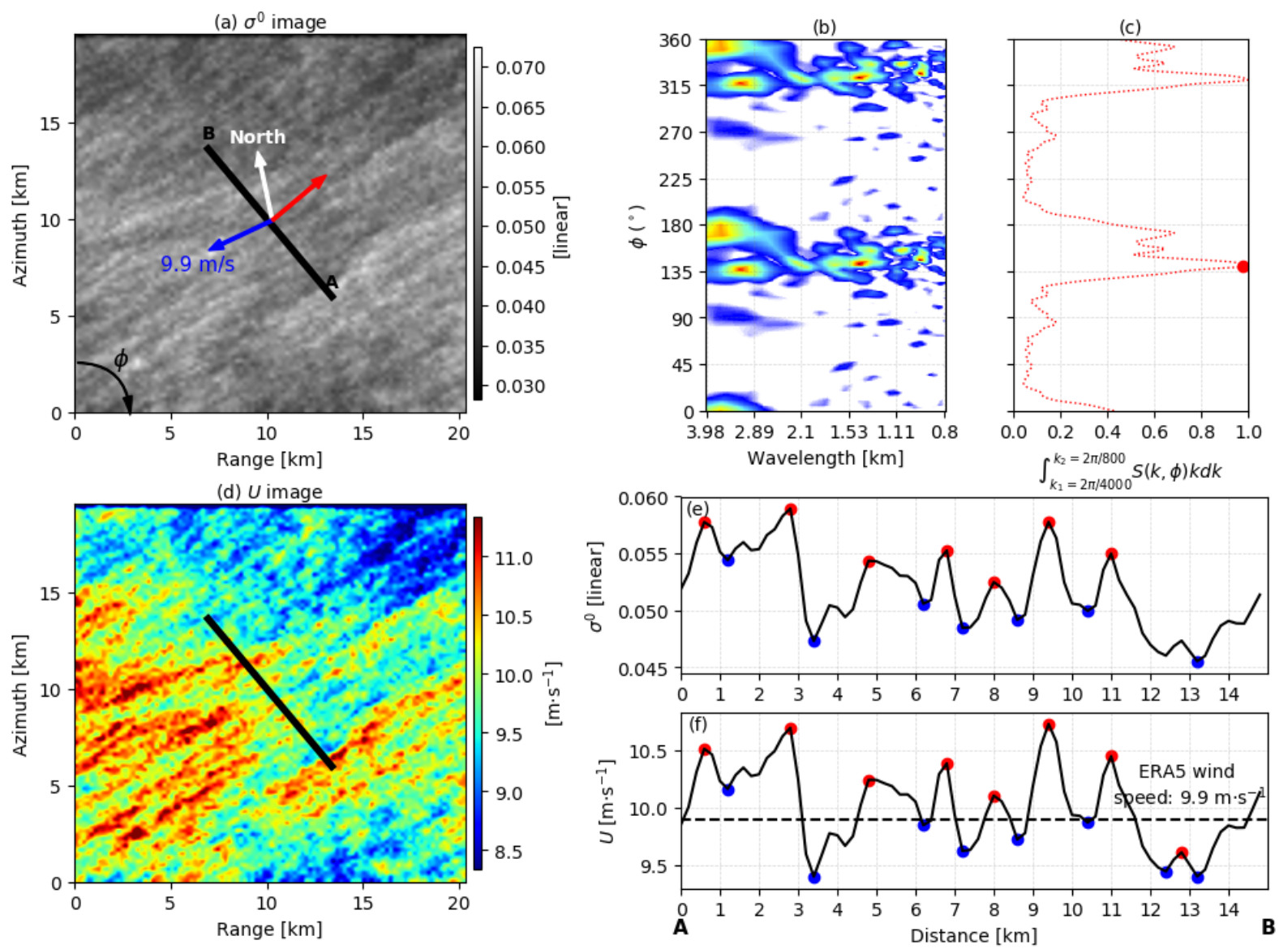

Fig. 2. Analysis of roll modulations in S-1 WV sea surface roughness images. Panel (a) is a typical roll image after smoothing to a 200-m pixel resolution $\sigma^{0}$ image. White, blue and red arrows indicate North, the ERA5 wind direction and extracted wind streak orientation, respectively. (b) 2-D FFT spectrum, $S(k, \phi)$, of the full resolution image within the expected wavenumber range of MABL patterns ( $\lambda=0.8$ and $4 \mathrm{~km})$. Angle $\phi$ is in SAR image coordinates, i.e. clockwise rotating from the azimuth to range direction. (c) Integral of $\mathrm{S}$ at each $\phi$, maximum marked as a red dot. (d) SAR-estimated wind speeds derived using the C-SARMOD GMF. (e) $\sigma^{0}$ transect profile along the black scan line in (a): from A to B. The profile (dashed line) has been smoothed with a $1 \mathrm{~km}$ length Hanning window. Red and blue dots indicate the detected local maximum and minimum. (f) similar to (e) but for SAR-retrieved local wind speed U.

Fig. 2 illustrates the process used to extract the roll-induced NRCS modulation from each WV roll imagette. The full resolution $\sigma^{0}$ image is box averaged to $200 \mathrm{~m}$ as shown in Fig. 2 (a), in order 
to filter out most of the ocean swell features. The chosen $200 \mathrm{~m}$ scale follows recommendations from previous studies (Koch, 2004; Horstmann and Koch, 2005; Christiansen et al., 2006). White and blue arrows in Fig. 2 (a) indicate North and the ERA5 10-meter wind direction, respectively.

An image modulation spectrum $S\left(k_{x}, k_{y}\right)$ is calculated using a 2-D FFT over the full resolution $\sigma^{0}$ image. The spectrum $S\left(k_{x}, k_{y}\right)$ is converted from Cartesian to Polar coordinates, $S(k, \phi)$. Fig. 2 (b) displays the partial spectrum in the $0.8-4.0 \mathrm{~km}$ wavelengths band. The angle $\phi$ is in SAR image coordinates. $\phi=0^{\circ}$ is in the increasing SAR azimuth direction along the satellite heading. $\phi=90^{\circ}$ is in the increasing SAR range direction (S-1 looks to the right). Since there is a $180^{\circ}$ direction ambiguity in wind streak orientation, the spectral energy peak maximises near $\phi=140^{\circ}$ and $320^{\circ}$. Note that multiple peaks are visible, principally associated with irregularities in the main linear features throughout the image scene. Still a dominant azimuthal peak direction is apparent and similar FFT methodologies have been used to extract the dominant orientation of MABL rolls (Gerling, 1986; Mourad and Walter, 1996; Li et al., 2013; Huang et al., 2018). For the example case of Fig. 2, panel (c) shows the corresponding profile of $S(\phi)=\int_{k=2 \pi / 4000}^{k=2 \pi / 800} S(k, \phi) d k d \phi$ with the maximum shown as a red dot. The red arrow in Fig. 2 (a) defines this dominant roll direction $\phi_{W S}$ (with $180^{\circ}$ ambiguity).

For each image, an estimate of the roll-induced $\sigma^{0}$ modulation along a $15 \mathrm{~km}$ transect is extracted. This $\sigma^{0}$ transect is located at the center of SAR scene and normal to the dominant wind streak direction, as shown in Fig. 2 (a). Five parallel lines (1 km width) of the backscatter are averaged and then smoothed using a Hanning window. As depicted in Fig. 2 (e), quasi-periodic $\sigma^{0}$ variations along the cross-roll transect are shown. The local maxima (red dots) and minima (blue dots) correspond to the alternating bright and dark bands on the backscatter image. The distance between adjacent bright or dark (roll wavelength) varies from 1 to $3 \mathrm{~km}$. It reveals local irregular roughness modulations with local changes of the surface wind intensity and/or direction, consistent with the multiple peaks present in the 2-D image spectrum. The modulation depth is defined as the difference between the mean bright $\left(\sigma_{B}^{0}\right)$ and dark $\left(\sigma_{D}^{0}\right)$ NRCS. The relative modulation depth, or contrast, is obtained after normalization by their average $\left(\sigma_{B}^{0}+\sigma_{D}^{0}\right) / 2$.

As shown in Fig. 2 (d), fine-scale $(\sim 200 \mathrm{~m})$ surface wind speed is also retrieved from each down-sampled WV $\sigma^{0}$ imagette using the C-SARMOD GMF (Mouche and Chapron, 2015). Here we used the co-located ERA5 wind direction instead of the extracted roll direction as input to the GMF. Similar to above, a SAR-retrieved wind speed (U) cross-roll modulation transect is extracted and smoothed in Fig. 2 (f). The obtained wind variations range from $0.5-1.5 \mathrm{~m} \cdot \mathrm{s}^{-1}$.

In summary, the following parameters relevant to roll-induced impacts on sea surface rough- 
ness are extracted from each CMwv-identified MABL roll WV SAR scene:

1) $\phi_{W S}\left[{ }^{\circ}\right]$ : Roll orientation with $180^{\circ}$ ambiguity in image coordinates: clockwise rotating from azimuth to range.

2) $\sigma_{B}^{0}$ [linear]: Mean NRCS for brightest roll modulation peaks.

3) $\sigma_{D}^{0}$ [linear]: Mean NRCS for the darkest roll modulation troughs.

4) $d \sigma^{0}=\sigma_{B}^{0}-\sigma_{D}^{0}$ [linear]: Modulation depth.

5) $d \sigma^{0} / \sigma^{0}=d \sigma^{0} /\left[\left(\sigma_{B}^{0}+\sigma_{D}^{0}\right) / 2\right]$ [linear]: Roll-induced NRCS perturbation.

6) $U_{B}\left[m s^{-1}\right]$ : Mean of the wind speed peaks.

7) $U_{D}\left[\mathrm{~ms}^{-1}\right]$ : Mean of the wind speed troughs.

8) $d U / U=\left(U_{B}-U_{D}\right) /\left[\left(U_{B}+U_{D}\right) / 2\right]$ : SAR-derived wind speed perturbation due to rolls.

\section{MABL roll occurrence rates}

\subsection{Occurrence statistics}

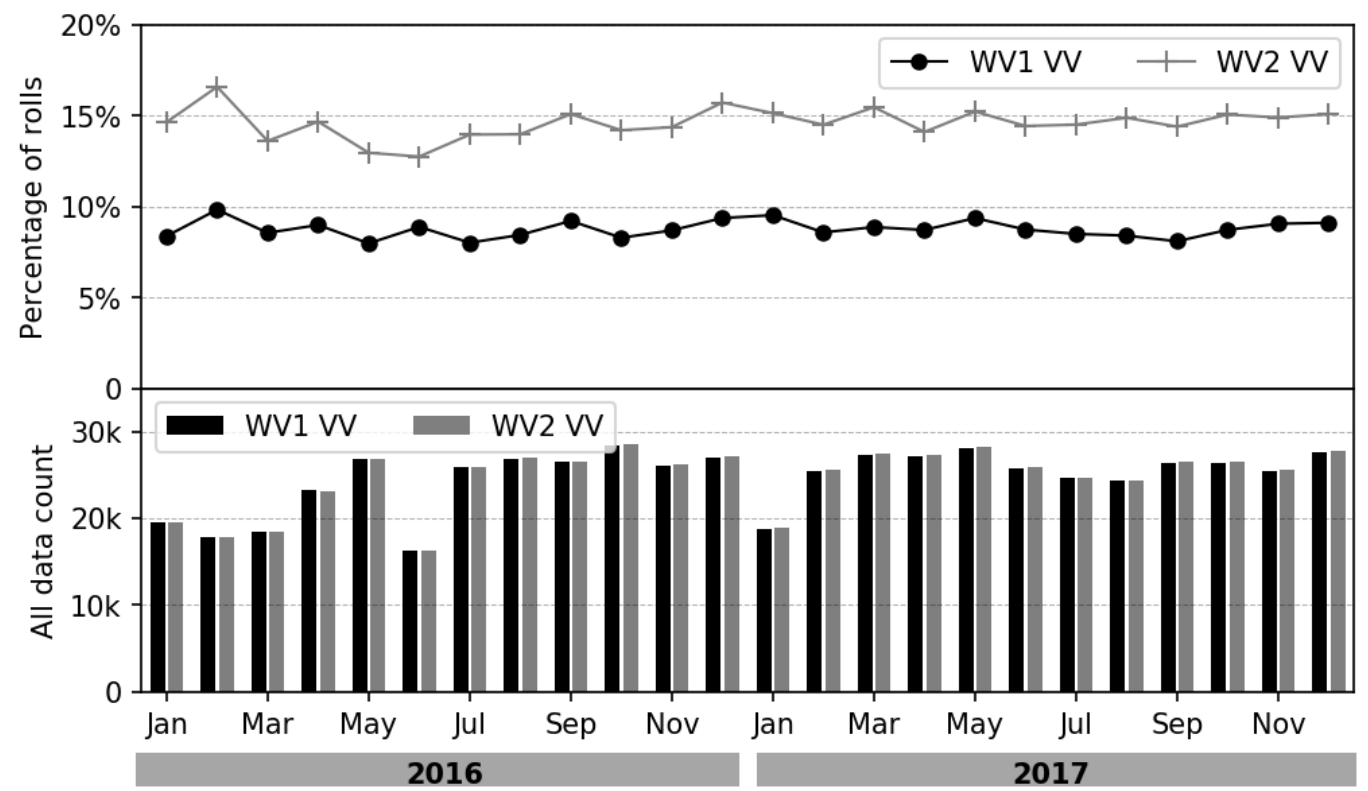

Fig. 3. Monthly statistics of detected roll events from all S-1A WV VV SAR imagettes. The top and bottom panels provide the percent-detected and the total number of imagettes examined in each month.

The automated classification considers a SAR imagette as a MABL roll event if clear evidence of organized quasi-linear wind streaks can be distinguished, and they dominate scene compared to other possible geophysical phenomena. Fig. 3 shows the percentage of MABL roll events 
relative to the total number of $\mathrm{WV}$ scenes acquired in each month. The image count per month of approximately 20k is about the same for WV1 and WV2. The overall fraction of identified roll events in WV2 is $\sim 15 \%$, while that for $\mathrm{WV} 1$ is $\sim 9 \%$ with no apparent monthly variability in roll frequency observed for either WV1 or WV2. The higher identification rate for WV2 compared to WV1 is likely related to higher visibility of the roll-induced wind speed changes at the larger incidence angle. The percentages of CMwv-classified roll events using the much smaller S-1B HH SAR dataset are approximately $11 \%$ and $6 \%$ for WV2 and WV1, respectively (not shown). Note, these detection rates are significantly lower than the reported average of $40-50 \%$ using visuallyselected images in coastal studies (Levy, 2001; Zhao et al., 2016).

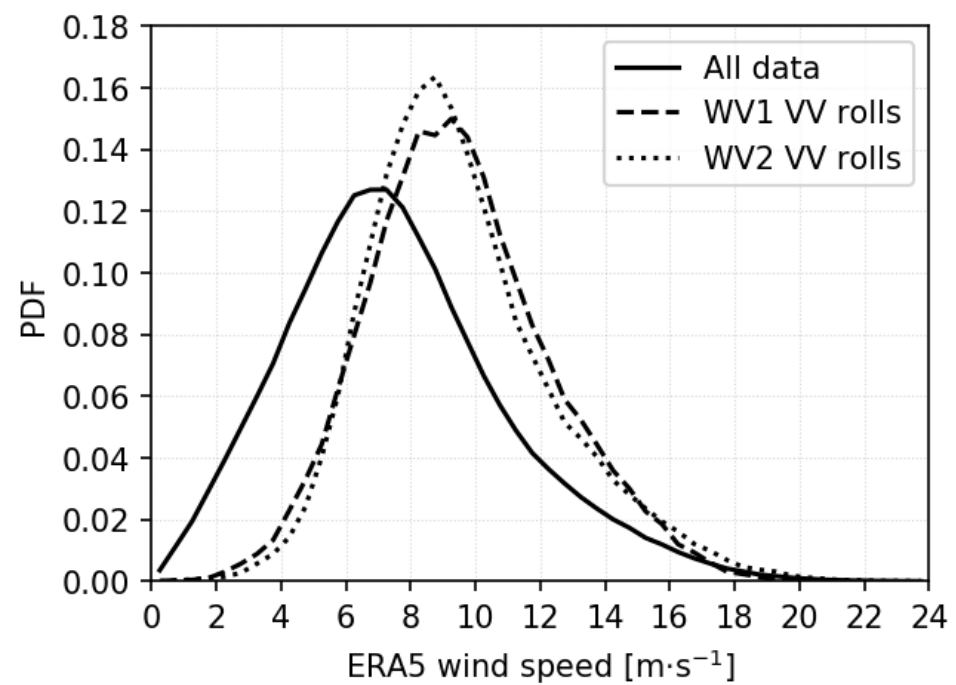

Fig. 4. Wind speed distributions of all S-1A WV VV SAR data and the CMwv-identified roll WV1 and WV2 data.

The probability density functions (PDFs) of the ERA5 surface wind speed when rolls were identified in the VV SAR data are shown in Fig. 4, along with the distribution for the entire S-1A WV dataset. The distributions for WV1 and WV2 roll events are similar. The most likely wind speed when rolls are identified is $9-10 \mathrm{~m} \cdot \mathrm{s}^{-1}$, which is higher than the $7 \mathrm{~m} \cdot \mathrm{s}^{-1}$ mode for the entire dataset. The low wind speed threshold for roll detection is near 2 to $3 \mathrm{~m} \cdot \mathrm{s}^{-1}$ for both WV1 and WV2. This is consistent with a postulated threshold of $3 \mathrm{~m} \cdot \mathrm{s}^{-1}$ (Weckwerth et al., 1997; Young et al., 2002; Zhao et al., 2016). The probability for roll detection rises rapidly with wind speed above this threshold. This is consistent with theoretical models that rolls usually form when shear production is an important aspect of the MABL dynamics (Brown, 1980; Etling and Brown, 1993). MABL roll identification versus wind speed and relative azimuth, which is the angle between 
the radar beam and surface wind direction, is shown in Fig. 5. Data are averaged in $20^{\circ}$ relative azimuth angle bins. Angles $0^{\circ}, 90^{\circ} / 270^{\circ}$ and $180^{\circ}$ are indicative of upwind, crosswind and downwind radar viewing directions. The bottom panel of Fig. 5 shows the S-1A WV sampling as a function of relative azimuth. Because S-1 SAR is right-side-looking and in a polar orbit, more data are acquired in upwind and downwind looks compared to crosswind due to the prevailing low-latitude easterlies and mid-latitude westerlies. However, thousands of roll images are collected at crosswind, which are mostly associated with the meridional flow around low and high pressure centers in the mid-latitudes.

Fig. $5(\mathrm{a}, \mathrm{b})$ show that, for wind speeds above $6 \mathrm{~m} \cdot \mathrm{s}^{-1}$, roll event detection rates are dramatically lower at crosswind for both incidence angles. Note that the roll detection rates for either WV1 or WV2 can reach 25-35\%, which are above the average $9 \%$ and $15 \%$ rates shown in Fig. 3 and are closer to the preciously reported detection rates (Levy, 2001; Zhao et al., 2016). While not shown, the detection rates of rolls from the smaller S-1B HH SAR dataset show similar behavior as $\mathrm{S}-1 \mathrm{~A} \mathrm{VV}$ for various wind speeds and relative azimuth. It is thus clear that the identification of roll events in S-1 SAR WV data depends strongly on all of incidence angle, wind speed and relative azimuth.

\subsection{Case study investigation}

To examine the MABL roll detection differences between WV1 and WV2, a section of Pacific Ocean $\left(15^{\circ} \mathrm{S}-30^{\circ} \mathrm{N}, 170^{\circ} \mathrm{E}-180^{\circ} \mathrm{E}\right)$ data is extracted from a descending S-1A pass on Feb 2, 2017 (Fig. 6). The SAR look direction is $287^{\circ}$ clockwise from North. Wind information from ERA5 shows that the wind field is fairly homogeneous at $9.5 \mathrm{~m} \cdot \mathrm{s}^{-1}$ and about $80^{\circ}$ direction in meteorological convention, so the relative azimuth is $333^{\circ}$. The central locations of the WV1 and WV2 imagettes reflect the standard leap-frog acquisition pattern. Concentrating on the $5^{\circ} \mathrm{N}$ to $18^{\circ} \mathrm{N}$ region, seven consecutive WV2 imagettes are classified as rolls. The atmospheric stability parameter $R i_{B}$ is slightly unstable at about -0.006 , which indicates that conditions are favorable for MABL roll development (Brown, 1980; Etling and Brown, 1993; Young et al., 2002). Thus, one would expect S-1 to observe clear roll imprints in both WV1 and WV2. However, only one WV1 case is classified as a roll event.

Fig. 6 shows the three pairs of WV1 and WV2 images that were acquired within a 2 minute span. WV2 images are displayed on the left column, and all these show clear periodic linear imprints of rolls. They have the same orientation, which is close to the ERA5 surface wind direction (blue arrows on images). In contrast, roll imprints are almost invisible on the three neighboring 


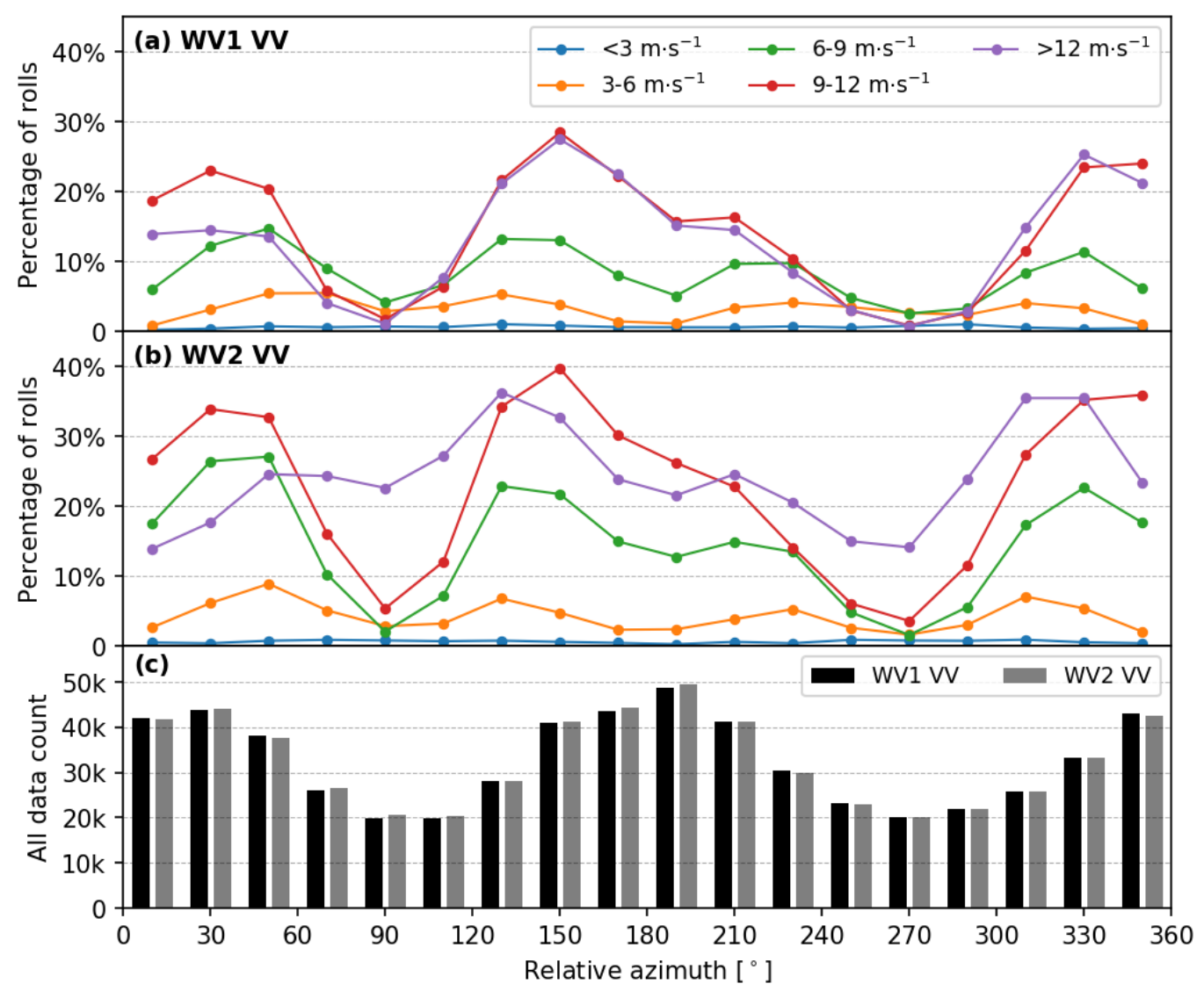

Fig. 5. Statistics of identified roll events from S-1A VV WV SAR images for different relative azimuth angles. (a) and (b) show the WV1 and WV2 percentages of identified rolls for selected wind speed ranges, and (c) gives the total of all images collected in each mode, respectively.

WV1 images shown in right column. Although one roll event was identified, the linear features on that image are weaker than those in the nearby WV2 images.

The computed image modulation parameters for these six cases are given in Table 1. The SAR-estimated wind speeds $U_{B}$ and $U_{D}$, corresponding to $\sigma_{B}^{0}$ and $\sigma_{D}^{0}$, are close to the mean ERA5 wind speed and approximately the same $d U$ levels are found in all six WV imagettes. However, the modulation depths, $d \sigma^{0}$, extracted from WV1 images are larger than that from WV2 data. When the modulation depth is normalized to contrast, $d \sigma^{0} / \sigma^{0}$, the three $\mathrm{WV} 2$ images have larger values than the three neighboring WV1 cases. This indicates that even though a smaller NRCS modulation is induced in WV2, it has a better roll detection capability than WV1 for the same wind conditions. Visually, roll imprints are more easily visible in WV2 than WV1 images as shown in Fig. 6. Roll detection is apparently sensitivity to the modulation contrast, which depends on the relative change in NRCS induced by small wind perturbation and the mean NRCS. These 


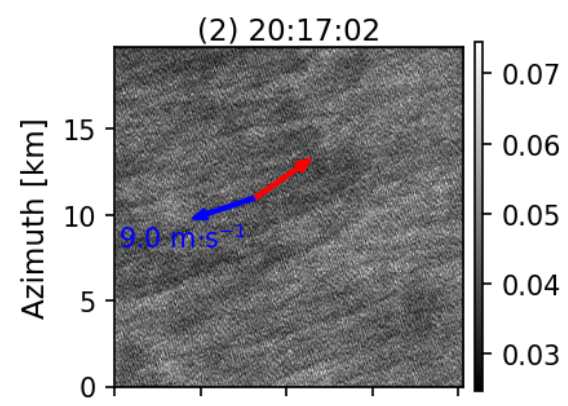

(4) $20: 17: 02$

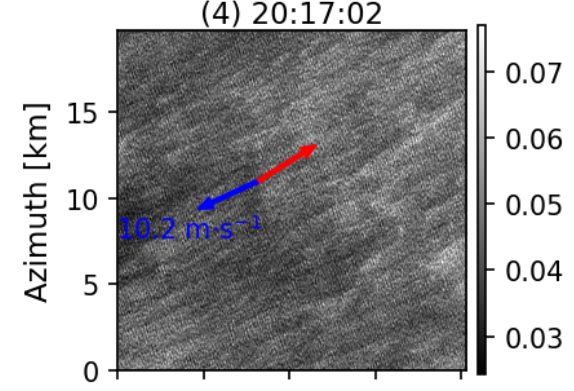

(6) $20: 17: 02$

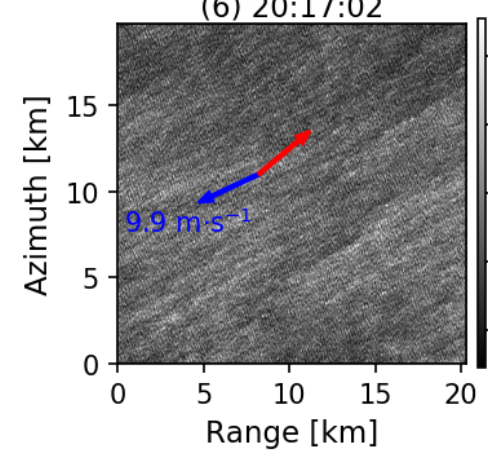

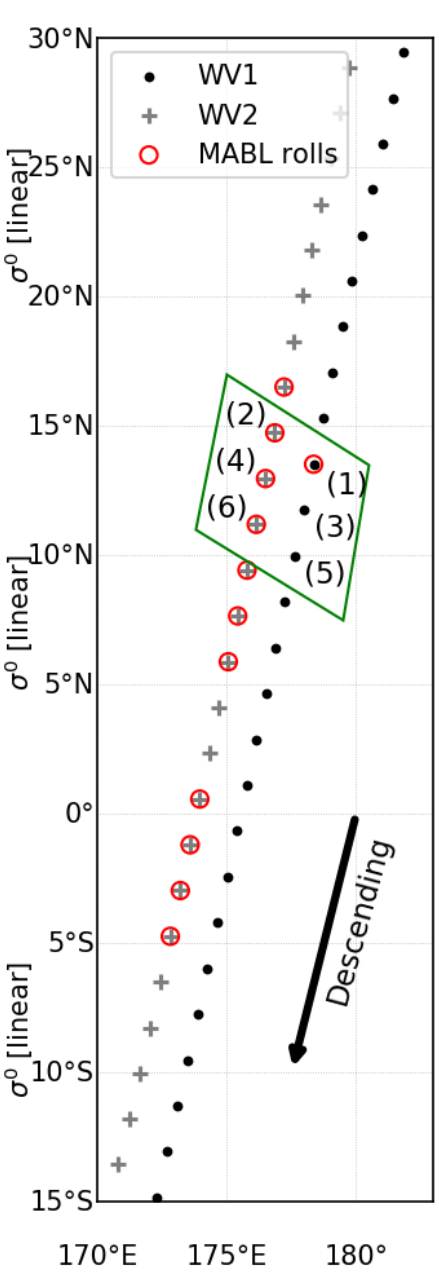

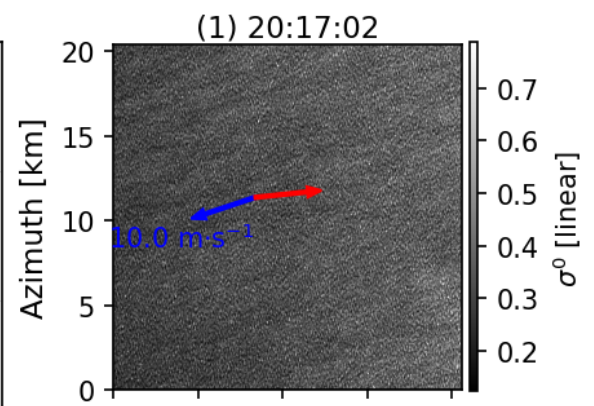

(3) $20: 17: 02$
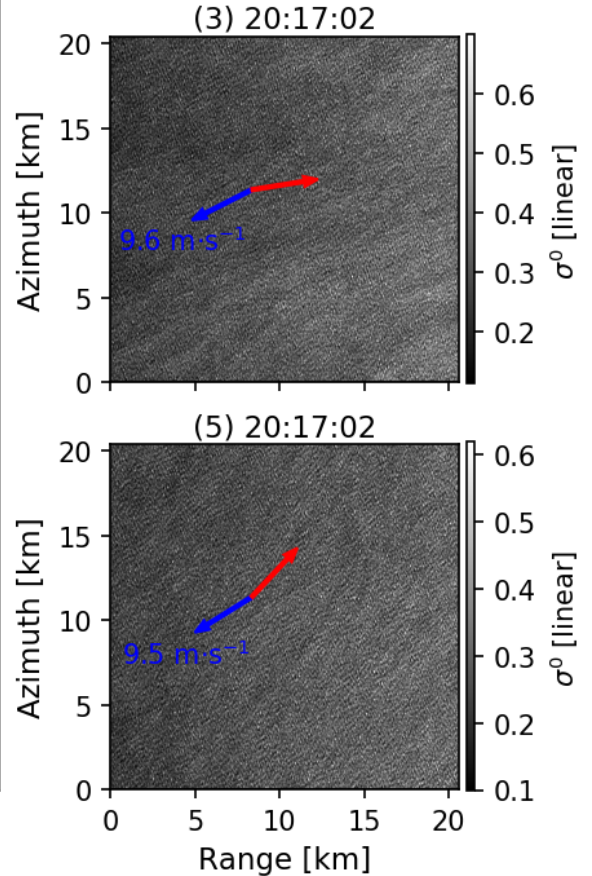

Fig. 6. Center panel shows center points of WV1 and WV2 acquisitions along an S-1A descending pass on 2017-02-02. Images identified by CMwv as roll events are shown with red circles. Three neighboring pairs WV2 and WV1 images (green box in the middle panel) are shown in the left and right panels. The blue and red arrows on the images indicate the ECMWF ERA5 surface wind and SAR backscatter-estimated roll directions, respectively.

two variables both vary as a function of wind speed, incidence and relative azimuth angles. Note, however, that the sole WV1 image classified as rolls has the least $d \sigma^{0} / \sigma^{0}$ among the six cases. It implies that the explanation for different roll detection rates between WV1 and WV2 is not simple. A statistical analysis of roll-related modulation parameters is thus necessary and given in Section 4. 
Table 1 Environmental variables and extracted roll modulation parameters for the six S-1A WV1 $\left(23^{\circ}\right)$ and WV2 $\left(36.5^{\circ}\right)$ image cases in Fig 6. $U 10$ and $\phi_{U 10}$ are the ERA5 10 meter wind speed and direction in meteorological convention. $R i_{B}$ is the atmospheric stability parameter estimated from the ERA5 variables. $\phi_{W S}^{\prime}$ is the extracted roll orientation in the same coordinate as $\phi_{U 10} . \sigma_{B}^{0}$ and $\sigma_{D}^{0}$ are the mean NRCS over roll-induced bright and dark on SAR images. $d \sigma^{0}, d \sigma^{0} / \sigma^{0}$ and $d U / U$ represent the roll-induced NRCS variation, relative NRCS variation (image contrast) and surface wind perturbation.

\begin{tabular}{|c|c|c|c|c|c|c|c|c|c|c|c|c|}
\hline Case ID & Swath & $\begin{array}{c}U 10 \\
{\left[\mathrm{~m} \cdot \mathrm{s}^{-1}\right]}\end{array}$ & $\begin{array}{c}\phi_{U 10} \\
{\left[{ }^{\circ}\right]}\end{array}$ & $\begin{array}{c}R i_{B} \\
{\left[\times 10^{-3}\right]}\end{array}$ & $\begin{array}{c}\phi_{W S}^{\prime} \\
{\left[^{\circ}\right]}\end{array}$ & $\begin{array}{c}\sigma_{B}^{0} \\
\text { [linear] }\end{array}$ & $\begin{array}{c}\sigma_{D}^{0} \\
{[\text { linear }]}\end{array}$ & $\begin{array}{c}d \sigma^{0} \\
\text { [linear] }\end{array}$ & $\frac{d \sigma^{0}}{\sigma^{0}}$ & $\begin{array}{c}U_{B} \\
{\left[\mathrm{~m} \cdot \mathrm{s}^{-1}\right]}\end{array}$ & $\begin{array}{c}U_{D} \\
{\left[\mathrm{~m} \cdot \mathrm{s}^{-1}\right]}\end{array}$ & $\frac{d U}{U}$ \\
\hline Fig 6 (1) & WV1 & 10.0 & 84 & -6.98 & 96 & 0.346 & 0.326 & 0.021 & 0.06 & 10.35 & 9.82 & 0.05 \\
\hline Fig 6 (3) & WV1 & 9.6 & 75 & -5.56 & 93 & 0.339 & 0.307 & 0.032 & 0.10 & 10.44 & 9.61 & 0.08 \\
\hline Fig $6(5)$ & WV1 & 9.5 & 70 & -6.99 & 55 & 0.302 & 0.278 & 0.024 & 0.08 & 9.54 & 8.99 & 0.06 \\
\hline Fig $6(2)$ & $\mathrm{WV} 2^{-}$ & $9.0^{-}$ & 84 & $-7.38^{-}$ & 67 & $0 . \overline{0} 51$ & 0.046 & $0 . \overline{0} \overline{5}$ & 0.10 & 9.58 & 9.05 & 0.06 \\
\hline Fig 6 (4) & WV2 & 10.2 & 77 & -6.29 & 70 & 0.053 & 0.047 & 0.006 & 0.12 & 10.09 & 9.39 & 0.07 \\
\hline Fig 6 (6) & WV2 & 9.9 & 77 & -6.36 & 62 & 0.056 & 0.049 & 0.007 & 0.12 & 10.34 & 9.68 & 0.07 \\
\hline
\end{tabular}

\section{S-1 WV NRCS response to roll imprints}

In this section, we take advantage of the large dataset to address the question of roll detection systematically. The parameters $d \sigma^{0}, d \sigma^{0} / \sigma^{0}$ and $d U / U$ are extracted from all the WV images that were identified as rolls by CMwv. The distributions of these quantities are binned as functions of ERA5 surface wind speed and relative azimuth for the different incidence angles and polarizations.

\subsection{Wind speed dependence}

The roll-induced modulation depth $d \sigma^{0}$ as a function of ERA5 wind speed for WV1 and WV2 in VV and HH polarization states are shown in Fig. 7 (a1,2). Box-plots are used to show the $d \sigma^{0}$ distribution in $2 \mathrm{~m} \cdot \mathrm{s}^{-1}$ bins from 3 to $19 \mathrm{~m} \cdot \mathrm{s}^{-1}$. Beyond this wind speed range, data are sparse particularly for $\mathrm{HH}$. For both incidence angles and polarizations, $d \sigma^{0}$ increases with wind speed. $\mathrm{WV} 1 d \sigma^{0}$ values are clearly larger than for WV2 for both VV and $\mathrm{HH}$ measurements. For winds larger than $13 \mathrm{~m} \cdot \mathrm{s}^{-1}, \mathrm{VV} d \sigma^{0}$ values exceed $\mathrm{HH} d \sigma^{0}$. These observations consistently follow the fact that the implied slope, $\partial \sigma^{0} / \partial U$, is on average larger at the lower incidence angle for both $\mathrm{VV}$ and $\mathrm{HH}$.

The SAR backscatter modulation contrast $d \sigma^{0} / \sigma^{0}$ is shown in Fig. 7 (b1,2). This roll-induced NRCS contrast is much less sensitive to surface wind speed than the modulation depth. One exception is the slightly larger values at low winds $\left(3-7 \mathrm{~m} \cdot \mathrm{s}^{-1}\right)$, particularly for $\mathrm{HH}$ data. This is likely because $\sigma^{0}$ can be very low in light winds even $d \sigma^{0}$ remains unchanged. A second observation is a likely roll identification threshold. For both WV1 and WV2 in either VV and $\mathrm{HH}$ polarization, the 10th percentile of $d \sigma^{0} / \sigma^{0}$ is almost constant near 0.04 . We hypothesize that this value corresponds to the effective roll detection floor for the end-to-end S-1 SAR WV and 

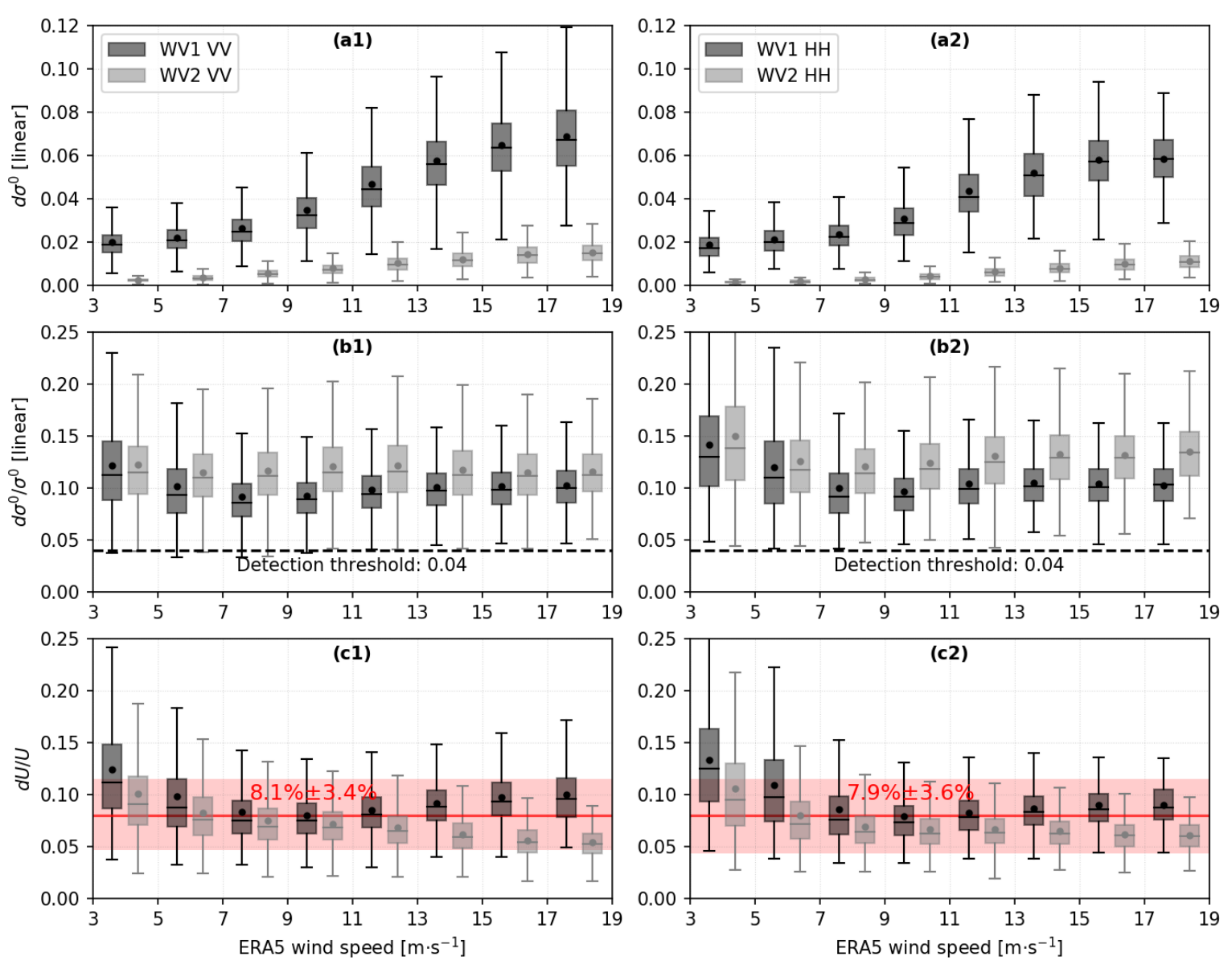

Fig. 7. Box plots of $d \sigma^{0}, d \sigma^{0} / \sigma^{0}$ and $d U / U$ for identified roll events from $\mathrm{WV} 1$ and $\mathrm{WV} 2$ in $\mathrm{VV}$ polarization (left panel) and $\mathrm{HH}$ (right panel). Statistics were conducted within each $2 \mathrm{~m} \cdot \mathrm{s}^{-1}$ bin from 3 to $19 \mathrm{~m} \cdot \mathrm{s}^{-1}$. Boxes indicate the 25th to 75th percentiles in each bin. Data mean and median are denoted using the point and line. The 10th and 90th percentiles are given by whiskers. The red line and shaded red in bottom panels represents the overall average and standard deviation of $d U / U$.

automated CMwv model system. Roll cases with image contrasts below this $4 \%$ level might be discernible by trained eyes, but by design, the non-supervised CMwv model was trained to only identify clearly visible and delineated roll cases (Wang et al., 2019b).

Referring again to Fig. 7 (b1,2), it is clear that WV2 $d \sigma^{0} / \sigma^{0}$ levels are generally higher than these for WV1 in both VV and HH measurements. This difference is consistent with the detection rate differences shown in Fig. 3 and Fig. 5. This indicates that roll-induced SAR backscatter contrast is greater at the higher incidence angle, which improves the chances of CMwv to identify roll events. Regarding polarization dependencies in the S-1 data, there is little $d \sigma^{0} / \sigma^{0}$ difference between VV and HH measurements. 

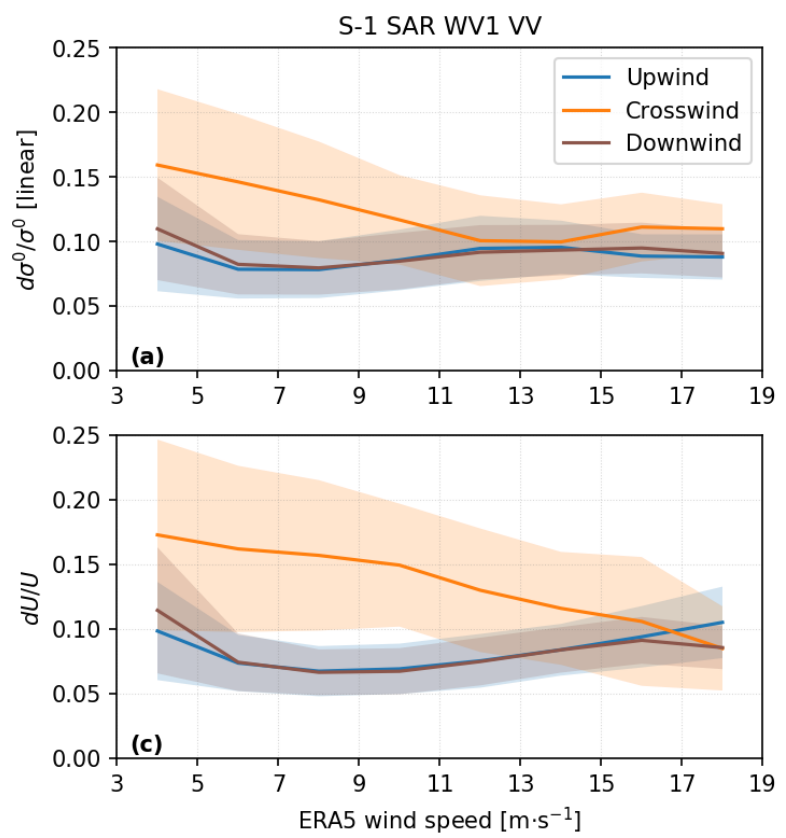
stronger (weaker) roll-induced wind perturbations.

\subsection{Dependence of roll detection on relative azimuth}

As noted earlier, ocean radar backscatter can be converted to wind speed using a GMF, which provides a means to normalize the results across the four WV mode incidence angle and polarization combinations. It also provides a geophysical quantification of MABL impact in terms of the surface wind speed perturbation. Global statistics of SAR-derived wind modulations $(d U / U)$ versus mean wind speed are shown in Fig. 7 (c1) and (c2). Similar to the $d \sigma^{0} / \sigma^{0}$ measurements, $d U / U$ mean values for WV1 and WV2 in VV and $\mathrm{HH}$ are relatively constant with wind speed. The average level of wind perturbation is $8 \%$ (standard deviation of $3.5 \%$ ). This value is consistent with previous field measurements of $7-10 \%$ obtained using low-level aircraft observations (Vandemark et al., 2001). The global ocean estimates show a slightly wider range of roll-induced wind perturbations, and the maximum wind perturbation seldom exceeds $15 \%$. For both VV and $\mathrm{HH}$, the $d U / U$ levels are slightly larger at low winds $\left(3-7 \mathrm{~m} \cdot \mathrm{s}^{-1}\right)$, which is similar to the $d \sigma^{0} / \sigma^{0}$ behavior. For wind speeds above $13 \mathrm{~m} \cdot \mathrm{s}^{-1}$, the detected rolls in WV1 (WV2) tend to be those with
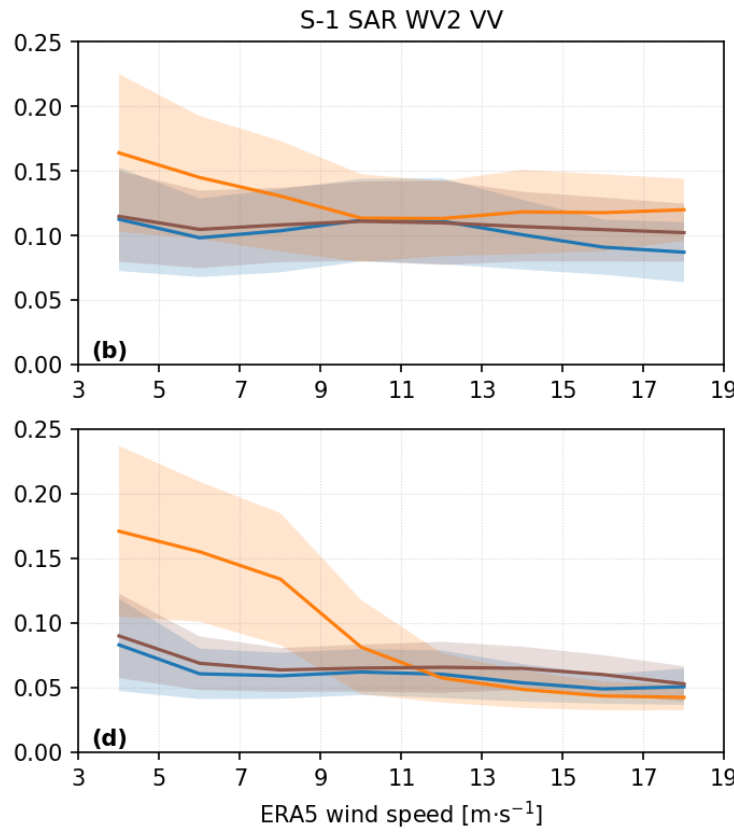

Fig. 8. Average $d \sigma^{0} / \sigma^{0}$ and $d U / U$ data from MABL roll events at up-, cross- and downwind SAR viewing angles as a function of wind speed. Data are from the 2016-2017 period with VV polarization. Statistics were computed for $\mathrm{a} \pm 15^{\circ}$ bin about the three relative azimuth angles, and within each $2 \mathrm{~m} \cdot \mathrm{s}^{-1}$ wind speed bin from 3 to $19 \mathrm{~m} \cdot \mathrm{s}^{-1}$. The line and shaded areas indicate the mean and one standard deviation.

For a given wind speed, MABL roll event data show that the observed SAR backscatter and 
wind speed perturbation estimates depend on the relative azimuth. Image contrast and wind perturbations within $\pm 15^{\circ}$ of the up-, cross- and downwind sectors for different wind speeds are shown in Fig. 8. Up- and downwind $d \sigma^{0} / \sigma^{0}$ and $d U / U$ are comparable for all wind speeds. When the SAR view is crosswind, both parameters markedly increase, particularly for WV1 measurements. For winds below $10 \mathrm{~m} \cdot \mathrm{s}^{-1}$, the identified crosswind roll events are associated with perturbation levels twice these for up- and downwind. These differences are largest at lower wind speeds. In terms of wind perturbation, detected crosswind roll events suggest a level twice that for up/down looks at speeds of 6-8 $\mathrm{m} \cdot \mathrm{s}^{-1}$. It is worth noticing that the limited S-1B HH SAR data show similar results with S-1A VV and thus not shown in the paper. The explanation for this is mostly a combination of roll dynamics, SAR sampling and image processing. Most of the crosswind WV images come from the flow around mid-latitude highs and lows and hence are in different thermal advection regimes, which are known to affect the rolls differently (Foster and Levy, 1998). However, about $25 \%$ of crosswind roll detection with the strongest relative perturbation strength occur at very low latitudes.

It is likely that this strong difference in directional radar sensitivity is related to short windwave variability and Bragg-scattering from these waves, which is polarization dependent. Fig. 9 shows $d \sigma^{0}$ distributions in $\mathrm{VV}$ and $\mathrm{HH}$ for both $\mathrm{WV} 1$ and $\mathrm{WV} 2$ at $9 \pm 1 \mathrm{~m} \cdot \mathrm{s}^{-1}$. As expected, the difference between polarizations is much more distinct at $36^{\circ}$ (WV2) than at $23^{\circ}$ (WV1), and with larger NRCS variability at VV than at $\mathrm{HH}$. The mean polarization difference (PD = $\sigma_{V V}^{0}-\sigma_{H H}^{0}$ ) is greater for WV2 observations (Quilfen et al., 1999; Kudryavtsev et al., 2013). This is consistent with the increasing impact of resonant small scatters that have short space-time relaxation scales, with increasing incidence angle (e.g. Mouche et al., 2007; Kudryavtsev et al., 2014). The differences between $d \sigma^{0}$ distributions at VV and $\mathrm{HH}$ are much less pronounced for crosswind configurations in the WV2 results.

Quantitatively, Fig. 9 shows that VV downwind $d \sigma^{0}$ levels are 3 times those of $\mathrm{HH} d \sigma^{0}$ at $36^{\circ}$ (WV2). This is consistent with the pure-Bragg wave scattering theory prediction of a four times VV-HH $\sigma^{0}$ difference according to typical scattering coefficient formulations (e.g. Eq. 3 and 4 in Kudryavtsev (2003)). For WV1, pure-Bragg wave growth predicts a factor of 1.75 between VV and $\mathrm{HH} \sigma^{0}$, which is consistent with the global downwind WV1 observations in Fig. 9. But at crosswind, the statistical distributions of $\mathrm{HH}$ and $\mathrm{VV} d \sigma^{0}$ are similar, and the mean levels are much lower than the along-wind data. So for crosswind, the dominant radar scattering mechanism must be almost scalar. That is because non-polarized scatters control the C-band radar-detected contrasts at crosswind. These waves are likely to be steeper intermediate scale $(10-50 \mathrm{~cm})$ gravity 
waves that require much stronger and longer duration wind forcing than for $\mathrm{cm}$-scale Bragg waves (Kudryavtsev, 2003; Kudryavtsev et al., 2014). The data then suggest that the roll-induced wind perturbations must be significantly enhanced when the S-1 SAR detects roll events in crosswind. This implication is that the crosswind roll detections are biased toward the strongest events.
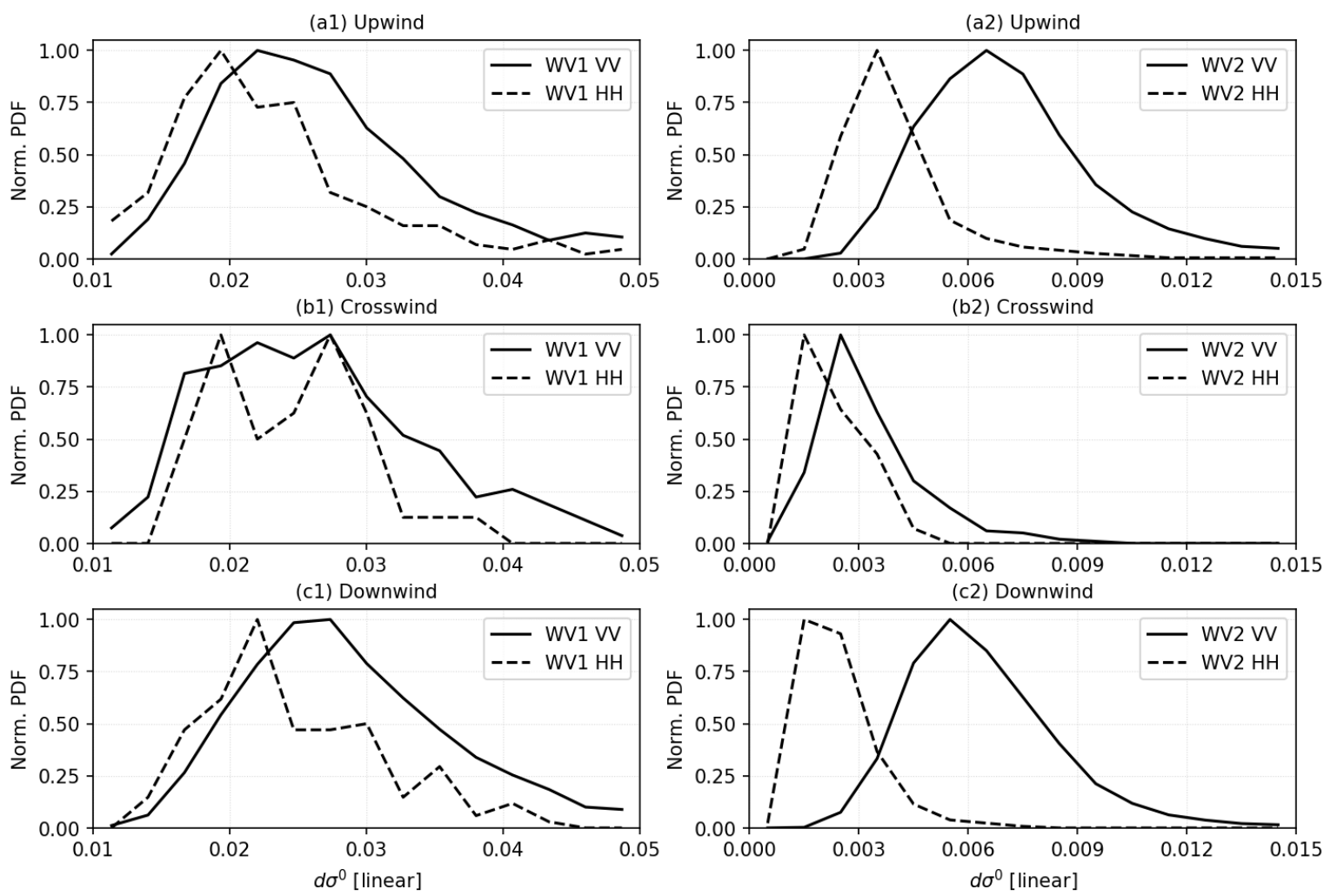

Fig. 9. $d \sigma^{0}$ distributions at up-, cross- and downwind $\left( \pm 15^{\circ}\right.$ bin) for WV1 (left panel) and WV2 (right panel) in VV and $\mathrm{HH}$ with wind speed of $9 \pm 1 \mathrm{~m} \cdot \mathrm{s}^{-1}$. Azimuth averaging is the same as for Fig. 8

\subsection{Comparison with C-band GMF simulations}

To further examine the C-band SAR NRCS response to rolls with respect to wind speed and relative azimuth, we interpret the $\mathrm{S}-1 \mathrm{WV} d \sigma^{0}$ measurements with a simple GMF simulation in the right column of Fig. 10. C-SARMOD calculations were performed assuming an 8\% roll-induced wind speed change $(d U / U)$ at each wind speed and direction for incidence angles of $23^{\circ}$ and $36.5^{\circ}$ over a wind speed range of 4.5 to $13.5 \mathrm{~m} \cdot \mathrm{s}^{-1}$ across the full range of relative azimuth angle.

Corresponding SAR measurement statistics are collected in $20^{\circ}$ relative azimuth and $3 \mathrm{~m} \cdot \mathrm{s}^{-1}$ wind speed bins (left column of Fig. 10). The modulation depth $d \sigma^{0}$ is largest at upwind and downwind. In light winds $\left(3-6 \mathrm{~m} \cdot \mathrm{s}^{-1}\right), \mathrm{WV} 1$ and WV2 $d \sigma^{0}$ are nearly constant for both VV and 

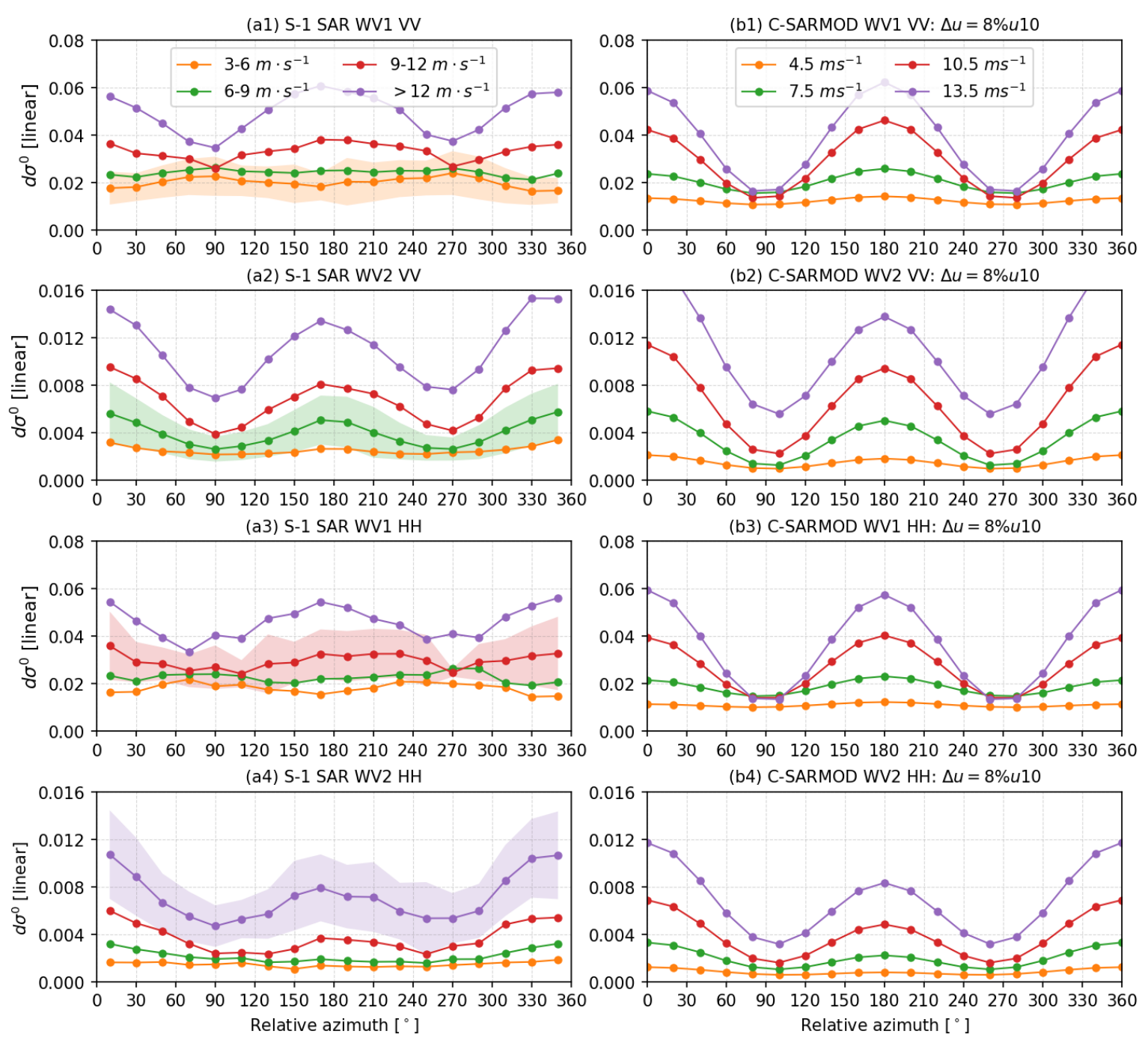

Fig. 10. Observed vs. predicted $d \sigma^{0}$ for roll events as function of wind speed and relative azimuth for WV1 and WV2 in VV and HH. Left panel are S-1 SAR WV results. Mean values are calculated for each $20^{\circ}$ relative azimuth angle. For clarity, estimates of the standard deviation (shaded) are only shown for one wind speed in each panel. The right panel provides the C-SARMOD simulations under an assumed $8 \%$ wind speed change due to roll impacts.

HH with WV1 around 0.02 and WV2 about 0.002 . With increasing wind speed, both VV and HH $d \sigma^{0}$ increase, with largest variations at up- and downwind.

The observed $d \sigma^{0}$ variations are generally similar, but there are some significant differences. In light winds $\left(3-6 \mathrm{~m} \cdot \mathrm{s}^{-1}\right), \mathrm{C}-\mathrm{SARMOD} d \sigma^{0}$ is also nearly direction independent, but at a lower magnitude than the SAR measurements. For moderate wind speeds, C-SARMOD predicts a stronger $d \sigma^{0}$ dependence on the relative azimuth than we observe. For instance, within the $9-12 \mathrm{~m} \cdot \mathrm{s}^{-1}$ wind 
speed range, $\mathrm{WV} 1 \mathrm{VV} d \sigma^{0}$ varies from 0.036 at upwind to about 0.026 at crosswind, and 0.037 at downwind. The corresponding C-SARMOD VV $d \sigma^{0}$ are $0.042,0.013$ and 0.046 , respectively. Similar discrepancies are found for other wind speed ranges. These differences between S-1 WV and C-SARMOD simulations are larger for WV1 than for WV2 in both VV and HH, and increase with increasing wind speed.

As might be expected from the results discussed in section 4.2, crosswind data show the largest differences with C-SARMOD. For WV1, the SAR $d \sigma^{0}$ is larger than 0.02 , and increases with wind speed. In contrast, the C-SARMOD $d \sigma^{0}$ is less than 0.02 , with no obvious wind speed trend. For WV2 at crosswind, both C-SARMOD and WV $d \sigma^{0}$ increase with wind speed, although CSARMOD increases less rapidly. It should be noted that the constant $8 \%$ wind modulation due to rolls will not be valid across the full scope of the model-data comparison.

\section{Discussion and Conclusions}

The combination of S-1 SAR WV data with automated image classification provides thousands of new MABL roll observations across most of the global ocean. Assessment results show that the $36^{\circ}$ incidence angle measurements have a clear benefit for MABL roll investigations and improve on wave mode data from the earlier ERS and Envisat SAR ocean missions. In particular, there is a nearly 50\% increase in event detection for WV2 compared to WV1. While more roll events are detected at the larger incidence angle, the two incidence angles show several important similarities for MABL roll remote sensing. First, they share a lower detection threshold near $4 \%$ in NRCS contrast at wind speeds from 3 to $19 \mathrm{~m} \cdot \mathrm{s}^{-1}$. Estimates of the roll-induced surface wind speed perturbations at both incidence angles fall in the range of 5-10\%. Finally, WV1 and WV2 SAR roll event detection rates are 3-10 times greater for up- and down-wind compared to crosswind (Fig. 5). These results generally hold for both VV and HH, although WV2 VV is slightly more sensitive than HH to wind streak signatures as seen in Figs. 7 and 9. It is thus apparent that the best option for S-1 SAR measurements of this process is the WV2 VV-pol configuration. These findings suggest that any ocean SAR investigations of MABL rolls should carefully consider the relative azimuth and SAR incidence angles.

The central geophysical explanation for the observed radar dependencies under MABL roll forcing appears to be the combination of surface wind stress impacts and adjustment to this forcing by highly directional short wind waves. As discussed in Section 4.2, roll imprints are best captured by WV2 VV in the up and downwind configurations. It demonstrates that the local $d \sigma^{0}$ changes due to the roll field are significantly polarized. Short-scale polarizing surface structures 
correspond to the $\mathrm{cm}$-scale Bragg waves. These waves have short relaxation times, and they quasiinstantaneously adjust to wind changes. Thus in the majority of cases, SAR-imaged rolls are likely to reflect this rapid adjustment in $\mathrm{cm}$-scale waves for $\mathrm{km}$-scale regions under the helical roll vortex field (Alpers and Brümmer, 1994; Young, 2000; Vandemark et al., 2001). Using a C-band radar GMF as a first-order model for this wind-wave adjustment, Fig. 7 (c1) and (c2) show that the global-averaged $8 \%( \pm 3.5)$ level for wind speed fluctuations does a reasonable job of explaining the roll-induced NRCS modulation depth. This appears to be a robust observation, valid across most wind speeds, and consistent with previous aircraft wind measurements showing a range of 7-10\% (Vandemark et al., 2001). Results also indicate that the strength of the coherent secondary circulation scales with the intensity of the mean flow. These satellite-derived estimates of surface perturbation magnitude may help guide analytical and numerical models of eddy-impacted boundary layers.

An explanation for the largest difference of MABL roll detection between up and crosswind SAR viewing angles (see Figs. 5 and 8 ) is more nuanced. It is asserted that the dominant crosswind NRCS modulation mechanism under roll forcing must come from changes in steeper intermediate scale breaking or nearly breaking surface scattering facets (Kudryavtsev, 2003; Kudryavtsev et al., 2013). This conclusion is consistent with VV and HH pol differences observed in Fig. 9. Accordingly, and particularly at lower incidence angles, roll-induced surface wind variations must be increasingly vigorous in magnitude and duration for wind streak detection under crosswind conditions, as seen in Fig. 8.

Looking forward, this discrepancy in roll-field detection may be related to other environmental conditions that accompany events classified as MABL rolls using CMwv. Because S-1 is polarorbiting and right-looking, roll observations at crosswind will be primarily associated with flows around mid-latitudinal lows and highs. These conditions are associated with particular thermal advection regimes that induce first-order modulations of the rolls. So, crosswind rolls present complication in both remote sensing and in geophysical interpretation. More generally, this reinforces the hypothesis that the forcing conditions needed to generate sufficient surface waves for wind streak detection may change, and be convolved with the SAR look direction and incidence angle. Further work is required to clarify this issue as it pertains to MABL roll process studies using S-1 data. Future investigations may also take advantage of these findings to focus on radar measurements under highly unstable atmospheric conditions, possibly using dual- and quad-polarized SAR measurements (Kudryavtsev et al., 2014, 2019; Fan et al., 2019).

It is certain that observed detection rates and thresholds depend to some extent on the per- 
formance of the CMwv automated image recognition algorithm. In the case of MABL rolls, the model was intentionally trained to find scenes that were clearly visible by eye. Given the consistent roll detection statistics and results in Fig. 7, this visibly-evident criterion corresponds to a $4 \%$ threshold in backscatter modulation. This implies that conditions with weaker, less visible, roll imprints are not captured in the present analysis and datasets. This limitation suggests that the overall percentage of occurrence rates for MABL rolls over the ocean seen in Figs. 3 and 5 represent conservative or lower-end estimates. In principle this may also affect the SAR-derived estimate of the low wind threshold for observed rolls $\left(\sim 2 \mathrm{~m} \cdot \mathrm{s}^{-1}\right)$ shown in Fig. 4, but this value is consistent with previous estimates from theory and observations (Etling and Brown, 1993; Weckwerth et al., 1997). CMwv model limitations are not expected to impact the study conclusions drawn above pertaining to radar sensitivity to MABL roll impacts with changing incidence angle, relative azimuth, or polarization.

These new S-1 WV observations open avenues for further studies. On one hand, the revealed differences in SAR sensitivity to waves generated by roll impacts, particularly for crosswind views, should be further investigated. This may lead to new approaches for identifying unstable conditions, and $R i_{B}$ retrieval methods. In that context, dual- and quad-polarized SAR observations (Fan et al., 2019), might be favored. In particular, short-scale polarized scatter contributions can be isolated to more precisely analyze local roll signatures. The growing number of quad-polarization observations, from Radarsat-2, Gaofen-3 and the new Radarsat Constellation Mission (RCM), are expected to serve this purpose. On the other hand, though the weak roll imprint cases are excluded in the present classified dataset, this S-1 SAR database is still state-of-the-art in terms of providing an overall global view of roll field characteristics (wavelength \& orientation) as well as the ability to relate these data to near-surface forcing from the tropics to high latitudes. This massive classified WV SAR images can thus be used to support boundary layer studies over the world's ocean to advance understandings of km-scale MABL coherent roll structures on turbulent momentum fluxes.

\section{Acknowledgements}

The authors would like to thank the anonymous reviewers for their constructive comments that improved the paper. This study is supported by ESA Sentinel-1 Mission Performance Center (4000107360/12/I-LG), ESA S1-4SCI Ocean Study (4000115170/15/I-SBo), and CNES TOSCA program (COWS) projects. Foster and Vandemark were supported by NASA Physical Oceanography grant NNX17AH17G. C. Wang is grateful for financial support from the China Scholarship 
Council (CSC) for his PhD, and the French ISblue project (ANR-17-EURE-0015) for this work.

\section{Appendix A. S-1 WV NRCS recalibration}

For each WV SAR image, ESA's Level-1 SLC product provides the digital number $D N=$ $\sqrt{I^{2}+Q^{2}}$ per pixel ( $I$ and $Q$ indicate the real and imaginary parts of SAR measurements). It can be used to compute the NRCS through radiometric calibration and noise correction:

$$
\sigma_{E S A}^{0}=D N_{i}^{2} / A_{i}^{2}-N E S Z \quad[\text { Linear }]
$$

where $A_{i}$ is the calibration lookup table (LUT) provided in the annotation files for each image pixel. NESZ is the noise equivalent $\sigma^{0}$ estimated from the mode of $D N_{i}^{2} / A_{i}^{2}$ histogram for wind speeds less than $1 \mathrm{~m} \cdot \mathrm{s}^{-1}$ (Li et al., 2019a,b). The NESZ is 0.0014 and 0.0062 for S-1A WV1 and WV2 in VV, and 0.0012 and 0.0040 for S-1B WV1 and WV2 in HH.
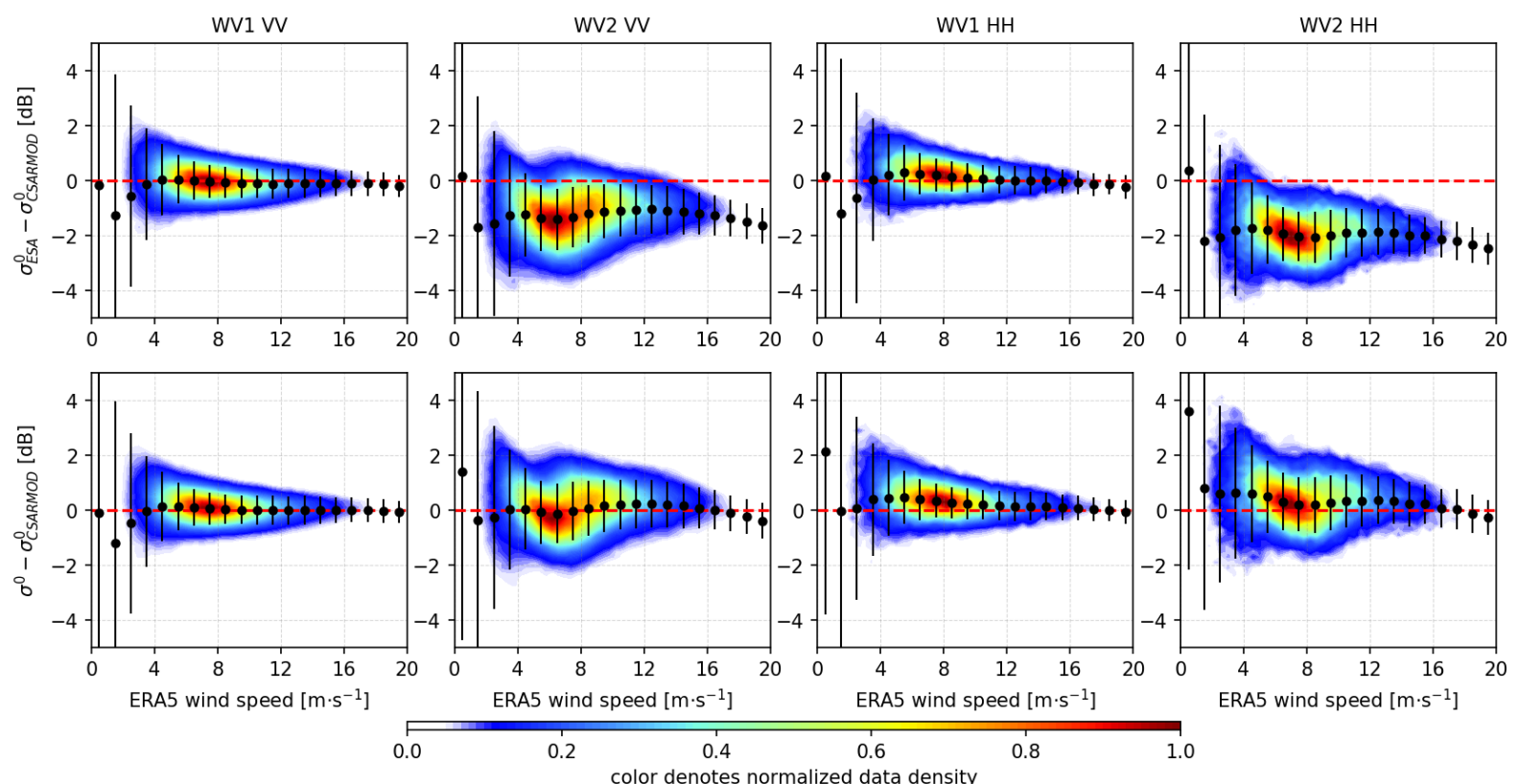

Fig. A.11. NRCS residual of pre- and post-recalibration (top and bottom) as function of wind speed for WV1 and WV2 in VV and HH. VV data are for S-1A in 2016-2017 and HH data are for S-1B from 15 Mar to $01 \mathrm{Jul}$ in 2017. Color denotes the normalized data density. The red dashed line indicates the $0 \mathrm{~dB}$ baseline. Black dots are the mean residual within $1 \mathrm{~m} \cdot \mathrm{s}^{-1}$ bin and the error bars represent one standard deviation. The $\sigma^{0}$ reference comes from the C-SARMOD model noted in the text. 
tions. NRCS comparisons between standard ESA WV data $\left(\sigma_{E S A}^{0}\right)$ and C-SARMOD predictions for WV1 and WV2 in VV and HH are given in the top panel of Fig. A.11. We then computed the mean $\sigma_{E S A}^{0}$ per image for WV data and fed the collocated ERA5 wind speed and direction as well as image center incidence angle into C-SARMOD to derive the expected $\sigma_{C-S A R M O D}^{0}$. Note that SAR data with mean $D N_{i}^{2} / A_{i}^{2}$ less than the NESZ are excluded. The error bar plots show the mean and 1st standard deviation within each $1 \mathrm{~m} \cdot \mathrm{s}^{-1}$ bin and the color denotes the normalized data density. It is clear that the NRCS residual $\left(\sigma_{E S A}^{0}{ }^{-} \sigma_{C-S A R M O D}^{0}\right)$ for WV1 data in both VV and $\mathrm{HH}$ is nearly $0 \mathrm{~dB}$ for all wind speeds. However, the WV2 NRCS residual has a nearly constant negative bias at all wind speeds of about $-1.6 \mathrm{~dB}$ for $\mathrm{VV}$ and $-2 \mathrm{~dB}$ for $\mathrm{HH}$. This is consistent with the NRCS assessment that discrepancies exist between S-1 WV data (after built-in calibration processing) and CMOD5.N predictions, particularly for measurements at the WV2 incidence angle of $36^{\circ}$ (Li et al., 2019b,a).

A recalibration constant $C_{O C}$ is calculated for each 12 day repeat cycle using C-SARMOD and ERA5 wind direction. This factor is then used to correct the WV NRCS as $\sigma^{0}=\sigma_{E S A}^{0} / C_{O C}$ in linear space. This procedure is applied for both WV1 and WV2 in VV and HH. The bottom panel of Fig. A.11 displays the NRCS residuals after recalibration. As expected, the WV2 mean NRCS values now lie within 0.1-0.2 $\mathrm{dB}$ of the C-SARMOD prediction for most wind speeds.

\section{References}

Alpers, W., Brümmer, B., 1994. Atmospheric boundary layer rolls observed by the synthetic aperture radar aboard the ERS-1 satellite. Journal of Geophysical Research 99, 12613. URL: http://doi.wiley.com/10.1029/ 94JC00421, doi:10.1029/94JC00421.

Alpers, W., Zhang, B., Mouche, A., Zeng, K., Chan, P.W., 2016. Rain footprints on C-band synthetic aperture radar images of the ocean - Revisited. Remote Sensing of Environment 187, 169-185. URL: https://linkinghub. elsevier.com/retrieve/pii/S003442571630387X, doi:10.1016/j.rse.2016.10.015.

Atkinson, B.W., Wu Zhang, J., 1996. Mesoscale shallow convection in the atmosphere. Reviews of Geophysics 34, 403-431. URL: http://doi.wiley.com/10.1029/96RG02623, doi:10.1029/96RG02623.

Babin, S.M., Sikora, T.D., Winstead, N.S., 2003. A Case Study of Satellite Synthetic Aperture Radar Signatures of Spatially Evolving Atmospheric Convection over the Western Atlantic Ocean. Boundary-Layer Meteorology 106, 527-546. URL: https : //doi.org/10.1023/A:1021236600569, doi:10.1023/A:1021236600569.

Bauer, P., Thorpe, A., Brunet, G., 2015. The quiet revolution of numerical weather prediction. Nature 525, 47-55. URL: http: //www.nature.com/articles/nature14956, doi:10.1038/nature14956.

Brilouet, P., Durand, P., Canut, G., 2017. The marine atmospheric boundary layer under strong wind conditions: Organized turbulence structure and flux estimates by airborne measurements. Journal of Geophysical Research: Atmospheres 122, 2115-2130. URL: https : //onlinelibrary.wiley.com/doi/abs/10.1002/2016JD025960, doi:10.1002/2016JD025960. 
Brown, R.A., 1980. Longitudinal instabilities and secondary flows in the planetary boundary layer: A review. Reviews of Geophysics 18, 683-697. URL: http://doi.wiley.com/10.1029/RG018i003p00683, doi:10.1029/RG018i003p00683.

Christiansen, M.B., Koch, W., Horstmann, J., Hasager, C.B., Nielsen, M., 2006. Wind resource assessment from C-band SAR. Remote Sensing of Environment 105, 68-81. URL: http://www.sciencedirect. com/science/article/pii/S0034425706002239https://linkinghub.elsevier.com/retrieve/pii/ S0034425706002239, doi:https://doi.org/10.1016/j.rse.2006.06.005.

Etling, D., Brown, R.A., 1993. Roll vortices in the planetary boundary layer: A review. Boundary-Layer Meteorology 65, 215-248. URL: http://link. springer.com/10.1007/BF00705527, doi:10.1007/BF00705527.

Fairall, C.W., Bradley, E.F., Hare, J.E., Grachev, A.A., Edson, J.B., 2003. Bulk Parameterization of AirSea Fluxes: Updates and Verification for the COARE Algorithm. Journal of Climate 16, 571-591. URL: http://journals. ametsoc.org/doi/abs/10.1175/1520-0442\{\%\}282003\{\%\}29016\{\%\}3C0571\{\%\}3ABPOASF \{\%\}3E2.0. CO $\{\%\} 3 B 2$, doi:10.1175/1520-0442 (2003) 016<0571:BPOASF>2 . 0 . CO; 2 .

Fan, S., Kudryavtsev, V., Zhang, B., Perrie, W., Chapron, B., Mouche, A., 2019. On C-Band Quad-Polarized Synthetic Aperture Radar Properties of Ocean Surface Currents. Remote Sensing 11, 2321. URL: https : / /www .mdpi . com/ 2072-4292/11/19/2321, doi:10.3390/rs11192321.

Foster, R.C., Levy, G., 1998. The Contribution of Organized Roll Vortices to the Surface Wind Vector in Baroclinic Conditions. Journal of the Atmospheric Sciences 55, 1466-1472. URL: http://journals. ametsoc.org/doi/abs/10.1175/1520-0469\%281998\%29055\%3C1466\%3ATCOORV\%3E2.0.CO\%3B2, doi:10. 1175/1520-0469(1998) 055<1466: TCOORV>2.0.CO;2.

Gerling, T.W., 1986. Structure of the surface wind field from the Seasat SAR. Journal of Geophysical Research 91, 2308. URL: http://doi .wiley.com/10.1029/JC091iC02p02308, doi:10.1029/JC091iC02p02308.

Glendening, J.W., 1996. Lineal Eddy Features under Strong Shear Conditions. Journal of the Atmospheric Sciences 53, 3430-3449. URL: http://journals.ametsoc.org/doi/abs/10. 1175/1520-0469\{\%\}281996\{\%\}29053\{\%\}3C3430\{\%\}3ALEFUSS \{\%\}3E2.0.CO\{\%\}3B2, doi:10.1175/ 1520-0469 (1996) 053<3430:LEFUSS $>2$. 0 . CO; 2.

Hein, P.F., Brown, R.A., 1988. Observations of longitudinal roll vortices during arctic cold air outbreaks over open water. Boundary-Layer Meteorology 45, 177-199. URL: http://link. springer. com/10.1007/BF00120822, doi:10.1007/BF00120822.

Horstmann, J., Koch, W., 2005. Measurement of Ocean Surface Winds Using Synthetic Aperture Radars. IEEE Journal of Oceanic Engineering 30, 508-515. URL: http://ieeexplore.ieee.org/document/1593798/, doi:10.1109/JOE. 2005.857514.

Huang, L., Li, X., Liu, B., Zhang, J.A., Shen, D., Zhang, Z., Yu, W., 2018. Tropical Cyclone Boundary Layer Rolls in Synthetic Aperture Radar Imagery. Journal of Geophysical Research: Oceans 123, 2981-2996. URL: http://doi.wiley.com/10.1029/2018JC013755, doi:10.1029/2018JC013755.

Jackson, C.R., Apel, J.R., Editors, 2004. Synthetic Aperture Radar Marine User's Manual. US Department of Commerce, National Oceanic and Atmospheric Administration, National Environmental Satellite, Data, and Information Serve, Office of Research and Applications.

Koch, W., 2004. Directional analysis of SAR images aiming at wind direction. IEEE Transactions on Geoscience and Remote Sensing 42, 702-710. URL: http://ieeexplore . ieee.org/document/1288365/, doi:10.1109/ 
TGRS . 2003. 818811.

Kudryavtsev, V., 2003. A semiempirical model of the normalized radar cross-section of the sea surface 1. Background model. Journal of Geophysical Research 108, 8054. URL: http://doi.wiley.com/10.1029/2001JC001003, doi:10.1029/2001JC001003.

Kudryavtsev, V., Kozlov, I., Chapron, B., Johannessen, J.A., 2014. Quad-polarization SAR features of ocean currents. Journal of Geophysical Research: Oceans 119, 6046-6065. URL: http://doi.wiley.com/10.1002/ 2014JC010173, doi:10.1002/2014JC010173.

Kudryavtsev, V.N., Chapron, B., Myasoedov, A.G., Collard, F., Johannessen, J.A., 2013. On Dual Co-Polarized SAR Measurements of the Ocean Surface. IEEE Geoscience and Remote Sensing Letters 10, 761-765. URL: http://ieeexplore.ieee.org/document/6377256/, doi:10.1109/LGRS.2012.2222341.

Kudryavtsev, V.N., Fan, S., Zhang, B., Mouche, A.A., Chapron, B., 2019. On Quad-Polarized SAR Measurements of the Ocean Surface. IEEE Transactions on Geoscience and Remote Sensing 57, 8362-8370. URL: https: //ieeexplore.ieee.org/document/8742586/, doi:10.1109/TGRS. 2019.2920750.

Kuettner, J., 1959. The Band Structure of the Atmosphere. Tellus 11, 267-294. URL: https://www . tandfonline . com/doi/full/10.3402/tellusa.v11i3.9319, doi:10.3402/tellusa.v11i3.9319.

LeCun, Y., Bengio, Y., Hinton, G., 2015. Deep learning. Nature 521, 436-444. URL: http://www. nature.com/ doifinder/10.1038/nature14539, doi:10.1038/nature14539, arXiv: arXiv:1312.6184v5.

LeMone, M.A., 1973. The Structure and Dynamics of Horizontal Roll Vortices in the Planetary Boundary Layer. Journal of the Atmospheric Sciences 30, 1077-1091. URL: http://journals.ametsoc.org/doi/abs/ 10.1175/1520-0469\%281973\%29030\%3C1077\%3ATSADOH\%3E2.0.CO\%3B2, doi:10.1175/1520-0469(1973) $030<1077$ : TSADOH $>2.0 . \mathrm{CO} ; 2$.

Lemone, M.A., 1976. Modulation of Turbulence Energy by Longitudinal Rolls in an Unstable Planetary Boundary Layer. Journal of the Atmospheric Sciences 33, 1308-1320. URL: http://journals . ametsoc . org/doi/abs/ 10.1175/1520-0469\%281976\%29033\%3C1308\%3AMOTEBL\%3E2.0.CO\%3B2， doi:10.1175/1520-0469(1976) $033<1308$ : MOTEBL $>2.0 . \mathrm{CO} ; 2$.

Levy, G., 2001. Boundary layer roll statistics from SAR. Geophysical Research Letters 28, 1993-1995. URL: http://doi.wiley.com/10.1029/2000GL012667, doi:10.1029/2000GL012667.

Li, H., Mouche, A., Stopa, J.E., 2019a. Impact of Sea State on Wind Retrieval From Sentinel-1 Wave Mode Data. IEEE Journal of Selected Topics in Applied Earth Observations and Remote Sensing 12, 559-566. URL: https: //ieeexplore. ieee.org/document/8637022/, doi:10.1109/JSTARS.2019.2893890.

Li, H., Mouche, A., Stopa, J.E., Chapron, B., 2019b. Calibration of the Normalized Radar Cross Section for Sentinel-1 Wave Mode. IEEE Transactions on Geoscience and Remote Sensing 57, 1514-1522. URL: https: //ieeexplore. ieee.org/document/8465988/, doi:10.1109/TGRS.2018.2867035.

Li, X., Zheng, W., Yang, X., Zhang, J.A., Pichel, W.G., Li, Z., 2013. Coexistence of Atmospheric Gravity Waves and Boundary Layer Rolls Observed by SAR*. Journal of the Atmospheric Sciences 70, 3448-3459. URL: http://journals.ametsoc.org/doi/abs/10.1175/JAS-D-12-0347.1, doi:10.1175/JAS-D-12-0347.1.

Li, X.M., Lehner, S., 2014. Algorithm for Sea Surface Wind Retrieval From TerraSAR-X and TanDEM-X Data. IEEE Transactions on Geoscience and Remote Sensing 52, 2928-2939. URL: http://ieeexplore.ieee.org/ document/6564437/, doi:10.1109/TGRS. 2013.2267780.

Lin, H., Xu, Q., Zheng, Q., 2008. An overview on SAR measurements of sea surface wind. Progress in Natural Sci- 
ence 18, 913-919. URL: http://www.sciencedirect.com/science/article/pii/S1002007108001755, doi:10.1016/j.pnsc.2008.03.008.

Mouche, A., Chapron, B., 2015. Global C-Band Envisat, RADARSAT-2 and Sentinel-1 SAR measurements in copolarization and cross-polarization. Journal of Geophysical Research: Oceans 120, 7195-7207. URL: http://dx.doi.org/10.1002/2015JC011149, doi:10.1002/2015JC011149.

Mouche, A.A., Chapron, B., Reul, N., 2007. A simplified asymptotic theory for ocean surface electromagnetic wave scattering. Waves in Random and Complex Media 17, 321-341. URL: http://www.tandfonline.com/doi/ abs/10.1080/17455030701230261, doi:10.1080/17455030701230261.

Mouche, A.A., Hauser, D., Kudryavtsev, V., 2006. Radar scattering of the ocean surface and sea-roughness properties: A combined analysis from dual-polarizations airborne radar observations and models in $\mathrm{C}$ band. Journal of Geophysical Research 111, C09004. URL: http://doi.wiley.com/10.1029/2005JC003166, doi:10.1029/2005JC003166.

Mourad, P.D., Thompson, D.R., Vandemark, D.C., 2000. Extracting fine-scale wind fields from synthetic aperture radar images of the ocean surface. Johns Hopkins APL Technical Digest (Applied Physics Laboratory) .

Mourad, P.D., Walter, B.a., 1996. Viewing a cold air outbreak using satellite-based synthetic aperture radar and advanced very high resolution radiometer imagery. Journal of Geophysical Research: Oceans 101, 16391-16400. URL: http://doi.wiley.com/10.1029/96JC01123, doi:10.1029/96JC01123.

Quilfen, Y., Chapron, B., Bentamy, A., Gourrion, J., El Fouhaily, T., Vandemark, D., 1999. Global ERS 1 and 2 and NSCAT observations: Upwind/crosswind and upwind/downwind measurements. Journal of Geophysical Research: Oceans 104, 11459-11469. URL: http://doi.wiley.com/10.1029/1998JC900113, doi:10.1029/ 1998JC900113.

Rowe, A.K., Houze, R.A., 2015. Cloud organization and growth during the transition from suppressed to active MJO conditions. Journal of Geophysical Research: Atmospheres 120. URL: https://onlinelibrary . wiley . com/ doi/abs/10.1002/2014JD022948, doi:10.1002/2014JD022948.

Shin, H.H., Hong, S.Y., 2013. Analysis of Resolved and Parameterized Vertical Transports in Convective Boundary Layers at Gray-Zone Resolutions. Journal of the Atmospheric Sciences 70, 3248-3261. URL: http: //journals . ametsoc.org/doi/abs/10.1175/JAS-D-12-0290.1, doi:10.1175/JAS-D-12-0290.1.

Sikora, T.D., Ufermann, S., 2004. Marine Atmospheric Boundary Layer Cellular Convection and Longitudinal Roll Vortices, in: Synthetic Aperture Radar Marine User's Manual. US Department of Commerce, National Oceanic and Atmospheric Administration, National Environmental Satellite, Data, and Information Serve, Office of Research and Applications. chapter 14, pp. 266-290.

Sikora, T.D., Young, G.S., Fisher, C.M., Stepp, M.D., 2011. A Synthetic Aperture RadarBased Climatology of Open-Cell Convection over the Northeast Pacific Ocean. Journal of Applied Meteorology and Climatology 50, 594-603. URL: http://journals.ametsoc.org/doi/abs/10.1175/2010JAMC2624.1, doi:10.1175/ 2010JAMC2624.1.

Szegedy, C., Vanhoucke, V., Ioffe, S., Shlens, J., Wojna, Z., 2016. Rethinking the Inception Architecture for Computer Vision, in: 2016 IEEE Conference on Computer Vision and Pattern Recognition (CVPR), IEEE. pp. 2818-2826. URL: http://ieeexplore . ieee.org/document/7780677/, doi:10.1109/CVPR.2016.308.

Torres, R., Snoeij, P., Geudtner, D., Bibby, D., Davidson, M., Attema, E., Potin, P., Rommen, B., Floury, N., Brown, M., Traver, I.N., Deghaye, P., Duesmann, B., Rosich, B., Miranda, N., Bruno, C., L'Abbate, M., 
Croci, R., Pietropaolo, A., Huchler, M., Rostan, F., 2012. GMES Sentinel-1 mission. Remote Sensing of Environment 120, 9-24. URL: https://linkinghub.elsevier.com/retrieve/pii/S0034425712000600, doi:10.1016/j.rse.2011.05.028.

Vandemark, D., Mourad, P.D., Bailey, S.A., Crawford, T.L., Vogel, C.A., Sun, J., Chapron, B., 2001. Measured changes in ocean surface roughness due to atmospheric boundary layer rolls. Journal of Geophysical Research: Oceans 106, 4639-4654. URL: http://doi.wiley.com/10.1029/1999JC000051, doi:10.1029/ 1999JC000051.

Wang, C., Mouche, A., Tandeo, P., Stopa, J., Longépé, N., Erhard, G., Foster, R., Vandemark, D., Chapron, B., 2018. Labeled SAR imagery dataset of ten geophysical phenomena from Sentinel-1 wave mode (TenGeoP-SARwv). URL: https://doi .org/10.17882/56796, doi:10.17882/56796.

Wang, C., Mouche, A., Tandeo, P., Stopa, J.E., Longépé, N., Erhard, G., Foster, R.C., Vandemark, D., Chapron, B., 2019a. A labelled ocean SAR imagery dataset of ten geophysical phenomena from Sentinel1 wave mode. Geoscience Data Journal 0, gdj3.73. URL: https://onlinelibrary.wiley.com/doi/abs/10.1002/gdj3. 73, doi:10.1002/gdj3.73.

Wang, C., Tandeo, P., Mouche, A., Stopa, J.E., Gressani, V., Longepe, N., Vandemark, D., Foster, R.C., Chapron, B., 2019b. Classification of the global Sentinel-1 SAR vignettes for ocean surface process studies. Remote Sensing of Environment 234, 111457. URL: https://www.sciencedirect.com/science/article/pii/ S0034425719304766?dgcid=author, doi:10.1016/j.rse.2019.111457.

Weckwerth, T.M., Wilson, J.W., Wakimoto, R.M., Crook, N.A., 1997. Horizontal Convective Rolls: Determining the Environmental Conditions Supporting their Existence and Characteristics. Monthly Weather Review 125, 505-526. URL: http://journals.ametsoc.org/doi/abs/10. 1175/1520-0493\{\%\}281997\{\%\}29125\{\%\}3C0505\{\%\}3AHCRDTE\{\%\}3E2.0.CO\{\%\}3B2, doi:10.1175/ 1520-0493 (1997) 125<0505:HCRDTE>2.0.CO; 2.

Weston, K.J., 1980. An observational study of convective cloud streets. Tellus 32, 433-438. URL: https://www . tandfonline.com/doi/full/10.3402/tellusa.v32i5.10598, doi:10.3402/tellusa.v32i5.10598.

Young, G., Sikora, T., Winstead, N., 2008. Mesoscale Near-Surface Wind Speed Variability Mapping with Synthetic Aperture Radar. Sensors 8, 7012-7034. URL: http://www.mdpi.com/1424-8220/8/11/7012, doi:10.3390/ s8117012.

Young, G.S., 2000. SAR signatures of the marine atmospheric boundary layer: Implications for numerical forecasting. Johns Hopkins APL Technical Digest (Applied Physics Laboratory) .

Young, G.S., Kristovich, D.A.R., Hjelmfelt, M.R., Foster, R.C., 2002. Rolls, Streets, Waves, and More: A Review of Quasi-Two-Dimensional Structures in the Atmospheric Boundary Layer. Bulletin of the American Meteorological Society 83, 997-1001. URL: http://journals.ametsoc.org/ doi/abs/10.1175/1520-0477\{\%\}282002\{\%\}29083\{\%\}3C0997\{\%\}3ARSWAMA \{\%\}3E2.3.CO\{\%\}3B2, doi:10. 1175/1520-0477 (2002) 083<0997:RSWAMA>2 . 3. CO; 2 .

Zecchetto, S., 2018. Wind Direction Extraction from SAR in Coastal Areas. Remote Sensing 10, 261. URL: http://www .mdpi . com/2072-4292/10/2/261, doi:10.3390/rs10020261.

Zhang, J.A., Katsaros, K.B., Black, P.G., Lehner, S., French, J.R., Drennan, W.M., 2008. Effects of Roll Vortices on Turbulent Fluxes in the Hurricane Boundary Layer. Boundary-Layer Meteorology 128, 173-189. URL: http: //link. springer.com/10.1007/s10546-008-9281-2, doi:10.1007/s10546-008-9281-2. 
Zhang, L.L., Zhang, L.L., Du, B., 2016. Deep Learning for Remote Sensing Data: A Technical Tutorial on the State of the Art. IEEE Geoscience and Remote Sensing Magazine 4, 22-40. URL: http: //ieeexplore.ieee.org/ document/7486259/, doi:10.1109/MGRS.2016.2540798.

Zhao, Y., Li, X.M., Sha, J., 2016. Sea surface wind streaks in spaceborne synthetic aperture radar imagery. Journal of Geophysical Research: Oceans 121, 6731-6741. URL: http://dx.doi.org/10.1002/2016JC012040, doi:10. 1002/2016JC012040.

Zhu, P., 2008. Simulation and parameterization of the turbulent transport in the hurricane boundary layer by large eddies. Journal of Geophysical Research 113, D17104. URL: http://doi . wiley . com/10 . 1029/2007JD009643, doi:10.1029/2007 JD009643.

\section{List of Figure Captions}

- Figure 1: Ocean SAR data coverage for S-1 study datasets, (a) S-1A in VV polarization in 2016-2017 and (b) S-1B in HH polarization, from 15 Mar to $01 \mathrm{Jul}$ in 2017. Color denotes the number of $\mathrm{WV}$ images within each $5^{\circ}$ by $5^{\circ}$ spatial bin. The total number of imagettes in these datasets is $1,182,540$ and 197,442 , respectively.

- Figure 2: Analysis of roll modulations in S-1 WV sea surface roughness images. Panel (a) is a typical roll image after smoothing to a $200-\mathrm{m}$ pixel resolution $\sigma^{0}$ image. White, blue and red arrows indicate North, the ERA5 wind direction and extracted wind streak orientation, respectively. (b) 2-D FFT spectrum, $S(k, \phi)$, of the full resolution image within the expected wavenumber range of MABL patterns $(\lambda=0.8$ and $4 \mathrm{~km})$. Angle $\phi$ is in SAR image coordinates, i.e. clockwise rotating from the azimuth to range direction. (c) Integral of $\mathrm{S}$ at each $\phi$, maximum marked as a red dot. (d) SAR-estimated wind speeds derived using the C-SARMOD GMF. (e) $\sigma^{0}$ transect profile along the black scan line in (a): from A to B. The profile (dashed line) has been smoothed with a $1 \mathrm{~km}$ length Hanning window. Red and blue dots indicate the detected local maximum and minimum. (f) similar to (e) but for SAR-retrieved local wind speed U.

- Figure 3: Monthly statistics of detected roll events from all S-1A WV VV SAR imagettes. The top and bottom panels provide the percent-detected and the total number of imagettes examined in each month.

- Figure 4: Wind speed distributions of all S-1A WV VV SAR data and the CMwv-identified roll WV1 and WV2 data. 
- Figure 5: Statistics of identified roll events from S-1A VV WV SAR images for different relative azimuth angles. (a) and (b) show the WV1 and WV2 percentages of identified rolls for selected wind speed ranges, and (c) gives the total of all images collected in each mode, respectively.

- Figure 6: Center panel shows center points of WV1 and WV2 acquisitions along an S-1A descending pass on 2017-02-02. Images identified by CMwv as roll events are shown with red circles. Three neighboring pairs WV2 and WV1 images (green box in the middle panel) are shown in the left and right panels. The blue and red arrows on the images indicate the ECMWF ERA5 surface wind and SAR backscatter-estimated roll directions, respectively.

- Figure 7: Box plots of $d \sigma^{0}, d \sigma^{0} / \sigma^{0}$ and $d U / U$ for identified roll events from WV1 and WV2 in VV polarization (left panel) and HH (right panel). Statistics were conducted within each $2 \mathrm{~m} \cdot \mathrm{s}^{-1}$ bin from 3 to $19 \mathrm{~m} \cdot \mathrm{s}^{-1}$. Boxes indicate the 25 th to 75 th percentiles in each bin. Data mean and median are denoted using the point and line. The 10th and 90th percentiles are given by whiskers. The red line and shaded red in bottom panels represents the overall average and standard deviation of $d U / U$.

- Figure 8: Average $d \sigma^{0} / \sigma^{0}$ and $d U / U$ data from MABL roll events at up-, cross- and downwind SAR viewing angles as a function of wind speed. Data are from the 2016-2017 period with VV polarization. Statistics were computed for a $\pm 15^{\circ}$ bin about the three relative azimuth angles, and within each $2 \mathrm{~m} \cdot \mathrm{s}^{-1}$ wind speed bin from 3 to $19 \mathrm{~m} \cdot \mathrm{s}^{-1}$. The line and shaded areas indicate the mean and one standard deviation.

- Figure 9: $d \sigma^{0}$ distributions at up-, cross- and downwind ( $\pm 15^{\circ}$ bin) for WV1 (left panel) and WV2 (right panel) in VV and $\mathrm{HH}$ with wind speed of $9 \pm 1 \mathrm{~m} \cdot \mathrm{s}^{-1}$. Azimuth averaging is the same as for Fig. 8

- Figure 10: Observed vs. predicted $d \sigma^{0}$ for roll events as function of wind speed and relative azimuth for WV1 and WV2 in VV and HH. Left panel are S-1 SAR WV results. Mean values are calculated for each $20^{\circ}$ relative azimuth angle. For clarity, estimates of the standard deviation (shaded) are only shown for one wind speed in each panel. The right panel provides the C-SARMOD simulations under an assumed $8 \%$ wind speed change due to roll impacts.

- Figure A1: NRCS residual of pre- and post-recalibration (top and bottom) as function of 
wind speed for WV1 and WV2 in VV and HH. VV data are for S-1A in 2016-2017 and HH data are for S-1B from 15 Mar to $01 \mathrm{Jul}$ in 2017. Color denotes the normalized data density. The red dashed line indicates the $0 \mathrm{~dB}$ baseline. Black dots are the mean residual within $1 \mathrm{~m} \cdot \mathrm{s}^{-1}$ bin and the error bars represent one standard deviation. The $\sigma^{0}$ reference comes from the C-SARMOD model noted in the text.

\section{List of Table Captions}

- Table 1: Environmental variables and extracted roll modulation parameters for the six S-1A WV1 $\left(23^{\circ}\right)$ and WV2 $\left(36.5^{\circ}\right)$ image cases in Fig 6. $U 10$ and $\phi_{U 10}$ are the ERA5 10 meter wind speed and direction in meteorological convention. $R i_{B}$ is the atmospheric stability parameter estimated from the ERA5 variables. $\phi_{W S}^{\prime}$ is the extracted roll orientation in the same coordinate as $\phi_{U 10} . \sigma_{B}^{0}$ and $\sigma_{D}^{0}$ are the mean NRCS over roll-induced bright and dark on SAR images. $d \sigma^{0}, d \sigma^{0} / \sigma^{0}$ and $d U / U$ represent the roll-induced NRCS variation, relative NRCS variation (image contrast) and surface wind perturbation. 